\title{
On the "Born" term used in thermodynamic models for electrolytes
}

Jean-Pierre Simonin ${ }^{1}$

Laboratoire PHENIX, CNRS, Sorbonne Université (Campus P.M. Curie), 4 Place Jussieu, Case 51, F-75005, Paris, France ${ }^{\mathrm{a}}$

In the literature, many expressions for the Helmholtz or Gibbs energy of electrolyte solutions have included a term that takes into account the variation of the solution permittivity with the composition of solution (within, e.g., the SAFT formalism). This contribution is often called the "Born" term because it was inspired by the classic expression established by Born to describe the solvation energy of an ion. The present work is an attempt to get more physical insight into this semi-empirical "Born" term. The way in which it has been used in the literature is briefly examined and its typical magnitude is evaluated. Next it is proposed to use the non-primitive mean spherical approximation (MSA) model to calculate the chemical potential of an ion in a solution composed of charged hard spheres (the ions) and dipolar hard spheres (the solvent). The cation and the anion are monovalent monoatomic ions of equal diameter. The dipoles have a different size, and mimic water molecules. The theoretical expressions for this model were found to fulfill the Gibbs-Duhem relation, which suggests that they are correct. A rescaled ion-dipole contribution is introduced, in a form that is suitable for inclusion in electrolyte models. It is compared with a "Born" term expressed in the same framework. It is found that the former is in general not well estimated by the latter. The two might even be of opposite signs in the case of ions of sufficiently small size.

Keywords: Electrolytes; non-primitive mean-spherical approximation (MSA); Born term; SAFT.

a)E-mail: jpsimonin@gmail.com 


\section{INTRODUCTION}

The modeling of the thermodynamic properties of aqueous, or mixed-solvent, electrolyte solutions and of their phase equilibria is of great importance in various areas of physical chemistry. It has applications in many fields such as industrial chemistry, food processing, biorefining, pharmacy, refrigeration, geochemistry, water treatment and desalination, oil industry, environmental chemistry (e.g., speciation),... Electrolytes also represent a challenge for the development of analytical models because of the variety and complexity of the interactions involved in these solutions, such as long-ranged interionic forces, polarization effects, hydrogen bonding, ion pairing,...

Various models have been developed in the liferature by considering the solvent (often water) as a continuous medium that manifests itself only through its dielectric constant. Such models are often termed as 'primitive models. This is the case of the Pitzer model that is extremely popular in the geochemical community. However, this framework is viewed as insufficient in various other areas because the solvent is not taken into account explicitly, with the consequence that its properties are not correctly described. For instance it becomes rather awkward to use in the case of mixed solvents or when thermal properties (e.g., dilution enthalpies) are considered.

Nonetheless, the deyelopment of analytic models including the solvent explicitly is a much more difficult task. Chemical engineering models have been proposed to describe ionic solutions $^{1}$, often based on the notion of local composition ${ }^{2}$ such as electrolyte-NRTL ${ }^{3,4}$, or UNIQUAC $^{5}$. Another class of models that has received much attention in the past decades is based on the statistical-associated fluid theory $(\mathrm{SAFT})^{6}$. This type of model is based on the first-order perturbation theory of Wertheim ${ }^{7}$, which theory may accommodate various types of association between species in solution (viz. ion pairing, chemical association, chains, hydrogen bonding, solvation). The inclusion of all interactions and effects arising in electrolyte solutions is still a significant challenge for analytic explicit-solvent models.

In general, this type of electrolyte model accounts for the effect of electrostatic interactions by adding dedicated contributions corresponding to the various forces between the species, namely ion-ion, ion-solvent and solvent-solvent interactions. The effect of ion-ion forces is described $^{3,4,8}$ through the use of a Debye-Hückel or mean spherical approximation (MSA) ${ }^{9}$ term. The effect of solvent-solvent forces is taken into account in SAFT models through 
the Wertheim association term. Lastly, the contribution from ion-solvent forces is often implicitly described by inserting a so-called "Born" term ${ }^{10-13}$, which has been proposed as an extension of the formula provided by Born to calculate ion solvation energies in solution.

It should be mentioned that a few SAFT models ${ }^{14-16}$ have used another approach. Instead, the non-primitive ion-dipole MSA model (ID-MSA) ${ }^{17-19}$ was employed in order to account at the same time for the effect of the three types of electrostatic interactions in aqueous solutions. In the ID-MSA model, water and the ions are represented as dipolar hard spheres and charged hard spheres, respectively.

Besides this exception, many models have accounted for the effect of ion-solvent forces by adding an independent "Born" term to the Helmholtz or Gibbs energy, or directly to the chemical potential of an ion. This has been the case of models based on the PengRobinson equation of state (EOS) ${ }^{8,20}$, on the cubic-plus-association (CPA) $\operatorname{EOS}^{21,22}$, on the Soave-Redlich-Kwong (SRK) $\mathrm{EOS}^{23}$, on the electrolyte-NRTL model ${ }^{3,24,25}$, and on SAFTtype equations ${ }^{10-13}$. Another case is the $I I+\mathrm{IW}$ model $^{26,27}$ in which the chemical potential of an ion comprises an ion-ion (II), and an ion-solvent (IW), interaction term. This model is developed in an implicit-solvent frame, though not at the McMillan-Mayer (MM) level at which ion-solvent interactions should not be introduced (only effective solvent-averaged ion-ion forces in the infinite dilute solution are involved in the MM framework ${ }^{28}$ ). One may note that a "Born" contribution has not always been included ${ }^{29}$ in the literature, and that it has also been sometimes intentionally discarded ${ }^{30}$.

Originally, the "Born" term seems to have been introduced for the first time by Cruz and Renon in $1978^{3}$ on the basis of the Debye and McAulay theory depicted in the book by Harned and Owen ${ }^{31}$. The latter theory had been developed for the description of "salting in" and "salting out" effects caused by electrolytes on the partitioning of neutral molecules, and the electrical work associated with the change in dielectric constant caused by an electrolyte (or a non-electrolyte) appeared incidentally in the development of the theory ${ }^{31}$. Anyhow the formula itself and its derivation were basically copied from the Born equation ${ }^{32}$.

The introduction of this term was motivated by two observations: the relative permittivity of an ionic solution, $\varepsilon_{\text {sol }}$, is experimentally known to vary (decrease) with salt concentration $^{33}$; the Born equation provides a way of estimating the electrostatic energy of an isolated ion placed in a (pure) solvent regarded as a dielectric continuum (the solvation energy of the ion). Then, supposedly, by assimilating an ionic solution to a dielectric con- 
tinuum of dielectric constant $\varepsilon_{\text {sol }}$, it was assumed that the Born equation could be applied equally to the case of an ion in a mixture of solvent molecules and ions.

However, it seems to the best knowledge of this author that, after having been introduced 40 years ago, the "Born" term has never been questioned per se, and that its validity has not been justified so far by any fundamental study. Its use seems to be taken for granted and, by the way, no other theoretical expression is available to replace it. Anyhow it should be recognized that the rationale behind this formula, viz. the assimilation of the medium surrounding an ion to a dielectric continuum having the permittivity of the solution, may appear questionable or at best of limited validity. Indeed, the range of ion-solvent forces that appreciably influence the ion-solvent chemical potential is likely to be of much shorter range than the typical distance beyond which the relative permittivity of solution starts to have a sound physical meaning (this point will be discussed below).

It is the purpose of this work to examine the "Born" term more closely. This is done mainly within the framework of the semi-restricted version of the ID-MSA model of Blum et al. ${ }^{17-19}$. This model has been found to overestimate the contribution from dipole-dipole interactions ${ }^{34,35}$. On the other hand, it has been shown to provide a satisfactory description of ion $^{35-37}$ and electron ${ }^{38}$ solyation in polar liquids, and of interactions between ions and dipolar solutes in molten salts ${ }^{39}$. Therefore, the ID-MSA model may be expected to give a good description of ion-solvent interactions in the present study.

The methodology/followed in this work to get more insight into the "Born" term is as follows. A model of ionic solution, as sketched in Figure 1, will be considered. This solution is composed of hard spherical equally-sized monovalent monoatomic ions, and hard spheres of a different size with a centrally embedded point dipole that are intended to mimic water molecules. It/will be assumed that its thermodynamic properties may be derived from the ID-MSA theory. The cation, the anion and the dipole will be denoted by ${ }^{+},{ }^{-}$, and $\mathrm{W}$, respectively.

First, suitable parameters will be determined for the dipolar solvent $\mathrm{W}$ so that it may represent water at $25^{\circ} \mathrm{C}$ and 1 atm in a reasonable way. Then, the semi-restricted iondipole MSA model will be solved for electrolyte solutions in which the cation and the anion have the same size. Crystallographic diameters will be taken to characterize the ions in practical applications. This way, the model will involve only two MSA parameters for the solvent molecules (namely their size and dipole moment). An expression will be derived for 


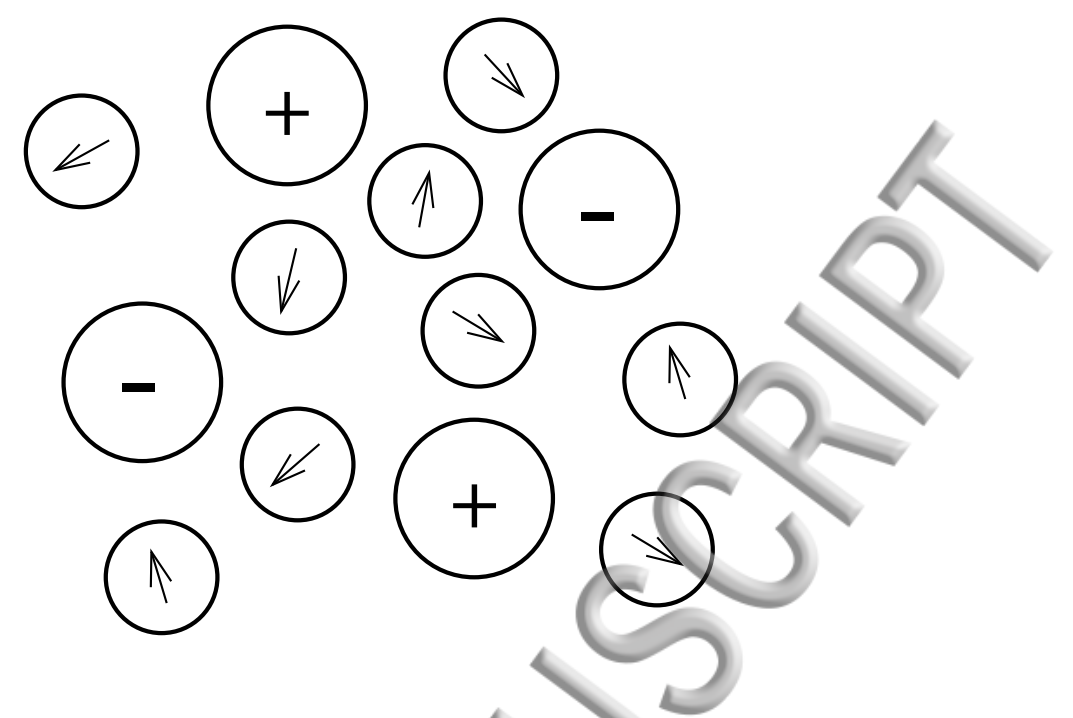

FIG. 1. Sketch of the ion-dipole mixture considered in this work, intended to model an aqueous electrolyte solution. The cation and the anion have the same hard core diameter, $\sigma_{i}$, and the dipolar hard spheres have a diameter $\sigma_{W}$.

a rescaled ion-dipole contribution to the chemical potential of an ion. This new contribution should be suitable for inclusion in usual models for electrolytes. This theoretical result will be compared with an expression for the "Born" term introduced at the same level of description.

The structure of this article follows these lines. The next section outlines the main features of the "Born" term as it has been used in the literature. Then the theoretical ingredients of this work and the formulas required to solve the ion-dipole MSA model are presented. It is shown that the chemical potentials for the salt and the solvent, obtained within the model, fulfill the Gibbs-Duhem relation, thus indicating that the formulas are correct. The appropriate expression for a rescaled ion-dipole contribution to the chemical potential of an ion is discussed. The third section is devoted to the presentation of the results. The magnitude of the "Born" term employed in the literature is briefly examined. Next, the ability of the "Born" term to describe the effect of ion-dipole interactions is scrutinized theoretically within the ID-MSA model. Finally, some additional remarks and prospects are given in the conclusion section. 


\section{BACKGROUND OF THE "BORN" TERM}

As has been said in the Introduction section, the "Born" term has been used in various types of expressions for the Helmholtz or Gibbs energy of an electrolyte solution. Hereafter we will only be concerned with aqueous solutions of salts composed of simple monovalent monoatomic ions (the case of multivalent ions is not well suited because of ion association effects that could not be treated adequately in the present framework).

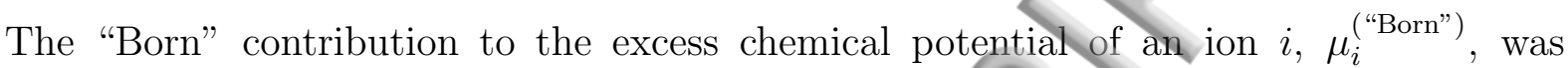
inspired from the Born equation ${ }^{32}$, which led to write ${ }^{3}$,

$$
\beta \mu_{i}^{(\text {"Born") }}=-\frac{\beta e^{2}}{4 \pi \varepsilon_{0} \sigma_{i}}\left(1-\frac{1}{\varepsilon_{\text {sol }}}\right) .
$$

in which $\beta=1 / k_{B} T$ ( $k_{B}$ is Boltzmann constant and $T$ is temperature), $e$ is the elementary charge, $\varepsilon_{0}$ is the permittivity of a vacuum, and $\sigma_{i}$ is the diameter of the simple (bare) ion. This equation expresses (in units of $k_{B} T$ ) the electrical energy of an ion in the solution relative to that in a vacuum. The energy of the ion was calculated by Born by summing the electrostatic energy density of the medium, $\varepsilon E^{2} / 2$ per volume unit (with $\varepsilon$ the permittivity of the medium and $E$ the electric field), in the whole space around the ion ${ }^{32}$.

Fundamentally, the "Born" term (Eq. (1)) was introduced as a simple way of accounting for the variation of ion-solvent interactions with salt concentration. The validity of Eq. (1) does not seem to have been analyzed up to now.

Application of Eq. (1) requires values for the relative permittivity of solution. This quantity has been measured for some aqueous electrolytes, mainly in the case of binaries at $25^{\circ} \mathrm{C}^{33}$. These experimental data do not seem to have been used generally in the literature (except in some cases ${ }^{26,27,40}$ ). Since the studies were done on temperature ranges at which data are scarce or unavailable, various estimates of $\varepsilon_{\text {sol }}$ have been utilized ${ }^{3,11,12}$. These estimates were obtained using, e.g., an equation proposed in 1973 by Pottel $^{41}$, or a formula introduced recently by Schreckenberg et al. ${ }^{11}$ in which $\left(\varepsilon_{\text {sol }}-1\right)$ is proportional to the concentration of water in the solution (see Supplementary Material for more details).

Once a dependence for the variation of $\varepsilon_{\text {sol }}$ with concentration has been adopted, the change of the chemical potential (Eq. (1)) with respect to its value at infinite dilution (the Born solvation energy) is,

$$
\beta \Delta \mu_{i}^{\text {("Born") }}=\frac{L_{0}}{\sigma_{i}}\left(\frac{1}{\varepsilon_{\text {sol }}}-\frac{1}{\varepsilon_{w}}\right),
$$


in which $\Delta$ denotes a difference w.r.t. infinite dilution, and $\varepsilon_{w}$ is the dielectric constant of water (denoted with a lower case $w$ ), and,

$$
L_{0}=\frac{\beta e^{2}}{4 \pi \varepsilon_{0}}
$$

which has the dimension of a length. One has $L_{0} \simeq 560.4 \AA$ at $25^{\circ} \mathrm{C}$.

It must be underlined that the "Born" contribution expressed by Eq. (2) is always positive because, as observed experimentally, $\varepsilon_{\text {sol }}$ is always smaller than $\varepsilon_{w}$

The magnitude of the mean ionic "Born" contribution to experimental values of $\ln \gamma_{s}$ (in which $\gamma_{s}$ is the mean salt activity coefficient taken from ref. 42) is

$$
\beta \Delta \mu_{s}^{(\text {("Born") }}=\frac{1}{2}\left[\beta \Delta \mu_{+}^{(\text {("Born") }}+\beta \Delta \mu^{\text {("Born") })}\right] .
$$

It is plotted in Figure 2 in the case of alkali chloride-solutions at $25^{\circ} \mathrm{C}$. It was calculated using Eq. (2) together with Shannon and Prewitt diameters for the $\sigma_{i}$ values $^{43}$, experimental data for $\varepsilon_{s o l}^{33,44}$, and $\varepsilon_{w}=78.4^{45}$. The salt concentration was calculated from the molality by using a parametrization of solution densities ${ }^{46}$.

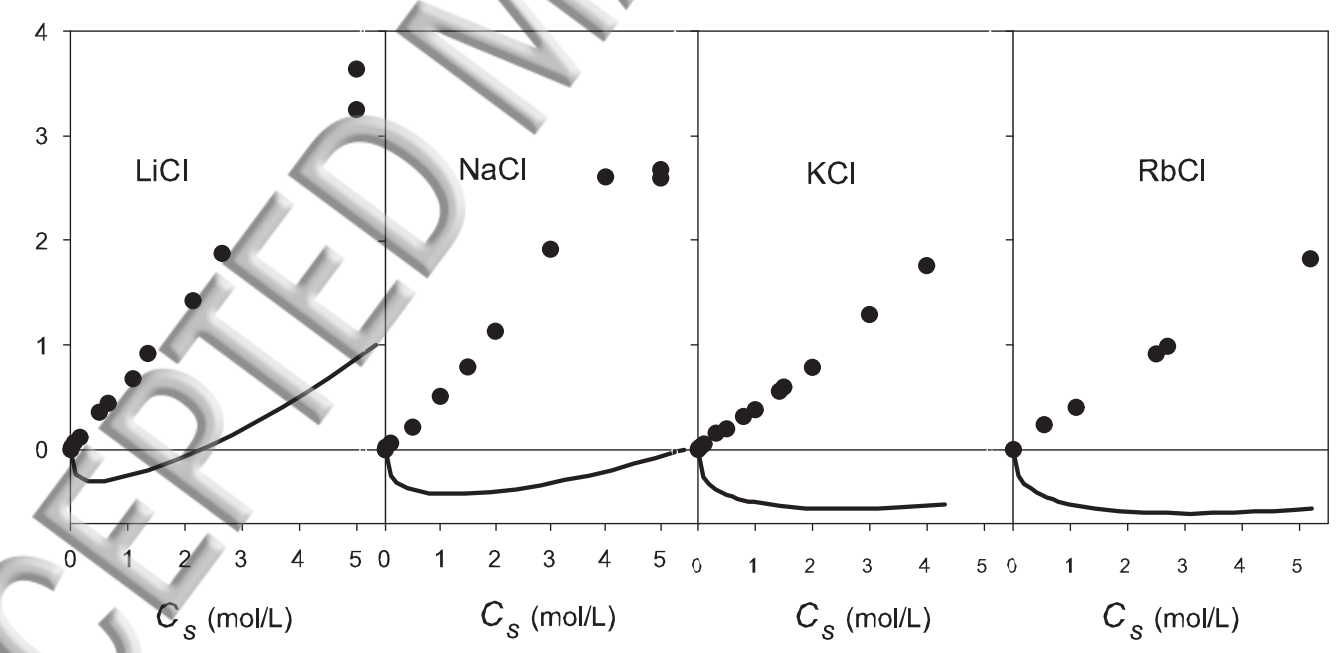

FIG. 2. Mean ionic "Born" contribution to $\ln \gamma_{s}$ (with $\gamma_{s}$ the mean salt activity coefficient) in the case of alkali chloride solutions (same scaling on the axes for the 4 salts): $(\bullet)$ Values for $\beta \Delta \mu_{s}^{\text {("Born") }}$ obtained using experimental data ${ }^{33,44}$ and Eq. (2); Solid lines $=$ Experimental values ${ }^{42}$ for $\ln \gamma_{s}$.

This figure shows that the mean ionic "Born" term, $\beta \Delta \mu_{s}^{\text {("Born") }}$, does not make a small contribution to $\ln \gamma_{s}$ in the case of alkali chloride solutions. In an electrolyte model, other 
terms in the expression of $\ln \gamma_{s}$ would have to compensate for this rather large contribution if (raw) experimental data for $\varepsilon_{\text {sol }}$ were used in Eq. (2). It is shown in the Supplementary Material addendum that, actually, smaller values for the "Born" term have been generally taken in the literature.

\section{THEORY}

\section{A. Basic relations in the ion-dipole MSA framework}

The system depicted in Figure 1 is a mixture of dipolar hard spheres of diameter $\sigma_{W}$ (the solvent, intended to mimic water) and oppositely charged hard spheres of equal diameter $\sigma_{i}$ (the ions). The moment of the embedded central point dipole is $m_{W}$. The number density (number of species per volume unit) of the 1-1 salt is $\rho_{s}\left(=\rho_{+}=\rho_{-}\right)$and that of the solvent is $\rho_{W}$. The subscript $s$ will be used to denote the salt.

The non-primitive ion-dipole MSA (ID-MSA) model was initially solved by Blum ${ }^{17}$ in the restricted case in which the ions and the dipoles have equal sizes, then in the semi-restricted case $^{18,19}$, and lastly in the general case where the species have arbitrary sizes ${ }^{18,34,47}$.

Hereafter we will use the semi-restricted version of the ID-MSA model, and we will mainly employ the notations of ref. 19. We now give the main formulas that are used to solve the model. Let us note that we will express the equations in terms of the parameters $b_{0}, b_{1}$ and $b_{2}$, which are functions of the ion-ion, ion-dipole, and dipole-dipole, correlation functions (see Eqs. (11)-(13) of ref. 19), respectively. We did not use the MSA screening parameter, $\Gamma$, and the polarization parameter, $\lambda$, because some of the equations may contain misprints. We note that formulas in terms of $b_{0}, b_{1}$ and $b_{2}$ have also been utilized previously by Liu et al. ${ }^{48}$ and by Herzog et al. ${ }^{16}$.

For a given ion-dipole mixture these 3 parameters are the unknowns to be determined first in order to compute the thermodynamic quantities. The equations of the model in the semi-restricted case involve two adimensional parameters,

$$
d_{0}^{2} \equiv 8 \pi \rho_{s} L_{0} \sigma_{i}^{2}
$$

for the ions, and

$$
d_{2}{ }^{2} \equiv \beta \frac{\rho_{W} m_{W}{ }^{2}}{3}
$$


for the dipolar solvent.

The three basic equations of the model $\operatorname{are}^{19}$,

$$
\begin{gathered}
a_{1}{ }^{2}+{a_{2}}^{2}=d_{0}{ }^{2}, \\
a_{1} K_{10}-a_{2}\left(1-K_{11}\right)=d_{0} d_{2}, \\
K_{10}{ }^{2}+\left(1-K_{11}\right)^{2}=y_{1}{ }^{2}+d_{2}{ }^{2},
\end{gathered}
$$

where

$$
\begin{gathered}
a_{1}=\frac{1}{2 D_{F}{ }^{2}}\left(\Delta-2 \beta_{6} D_{F}\right), \quad a_{2}=-\frac{b_{1}}{2 \beta_{6} D_{F}{ }^{2}}\left(\frac{\Delta}{2}+\frac{D_{F} \beta_{3}}{r}\right), \\
K_{10}=r \frac{b_{1}}{2 \Delta}\left(1+a_{1} \Lambda\right), \quad 1-K_{11}=\frac{1}{\Delta}\left(\beta_{3}-a_{2} b_{1} \Lambda \frac{r}{2}\right), \\
r=\sigma_{W} / \sigma_{i}, \\
\beta_{3}=1+b_{2} / 3, \quad y_{1}=\beta_{6} / \beta_{12}{ }^{2}, \\
\beta_{6}=1-b_{2} / 6, \quad \beta_{12}=1+b_{2} / 12, \quad \beta_{24}=1-b_{2} / 24, \\
\Delta=\frac{b_{1}{ }^{2}}{4}+\beta_{6}{ }^{2}, \quad D_{F}=\frac{1}{2}\left[\beta_{6}\left(1+b_{0}\right)-b_{1}{ }^{2} \frac{r}{12}\right], \quad \Lambda=\frac{1+b_{0}}{2}+\beta_{6} \frac{r}{6} .
\end{gathered}
$$

Let us mention that these equations were taken from ref. 19 without modification (except for the use of $r$ ), and that they are identical to those employed in previous works ${ }^{16,48}$.

The electrostatic contribution to the pressure, denoted by $P^{e l}$, is given by ${ }^{19}$,

$$
\beta P^{e l}=\frac{1}{12 \pi \sigma_{i}^{3}}\left[d_{0}^{2} b_{0}-4 \frac{d_{0} d_{2}}{r} b_{1}-6 \frac{d_{2}{ }^{2}}{r^{3}} b_{2}+J^{\prime}\right] \text {, }
$$

with

$$
\begin{aligned}
& J^{\prime}=\left(Q_{i i}^{\prime}\right)^{2}+\frac{1}{r}\left(1+\frac{1}{r}\right)\left(Q_{i d}^{\prime}\right)^{2}+\frac{1}{r^{3}}\left[\left(Q_{d d}^{\prime}\right)^{2}+2\left(q^{\prime}\right)^{2}\right] \\
& Q_{i i}^{\prime}=-a_{1}-2+\frac{\beta_{6}}{D_{F}} \quad Q_{i d}^{\prime}=\frac{b_{1}}{\Delta}\left[\beta_{3}+a_{1}\left(3 \Lambda-2 D_{F}\right)\right], \\
& Q_{d d}^{\prime}=\frac{2}{\Delta}\left[\beta_{3}^{2}-\frac{r}{2} b_{1} a_{2}\left(3 \Lambda-2 D_{F}\right)\right]-2, \quad q^{\prime}=\frac{\beta_{24}}{\beta_{12}^{2}} b_{2} .
\end{aligned}
$$

These relations were also taken from ref. 19. A misprint in the expression of $Q_{d d}^{\prime}(r$ instead of $1 / r$ ), and an obvious one in Eq. (104) of ref. 19 (opposite sign for $P$ ), were corrected, as was done before in refs 16 and 48. Let us note that a simpler expression for $P^{e l}$ is also available ${ }^{49}$ in the case of pure dipolar solvent. 
Expressions for the electrostatic contributions to the chemical potentials may be found in the paper of Blum and $\mathrm{Wei}^{18}$. By using Eq. (5) they may be written in the following more simple form,

$$
\beta \mu_{i}^{e l}=\frac{L_{0}}{\sigma_{i}} b_{0}-\frac{L_{0}}{\sigma_{W}} \frac{d_{2}}{d_{0}} b_{1}
$$

with $i=+$ or - because $\mu_{+}=\mu_{-}$for the present system in which the cation and the anion have the same size and charge in absolute value.

In Eq. (19) the first term,

$$
\beta \mu_{i}^{i i}=\frac{L_{0}}{\sigma_{i}} b_{0}
$$

represents the contribution of ion-ion interactions to $\beta \mu_{i}^{e l}$ (note that $b_{0}<0$ ), and the second term,

$$
\beta \mu_{i}^{i d}=-\frac{L_{0}}{\sigma_{W}} \frac{d_{2}}{d_{0}} b_{1}
$$

is the contribution of ion-dipole interactions. Therefore,

$$
\beta \mu_{i}^{e l}=\beta \mu_{i}^{i i}+\beta \mu_{i}^{i d}
$$

For the solvent one has ${ }^{48}$,

$$
\beta \mu_{W}^{e l}=-\frac{\left(m_{W}^{*}\right)^{2}}{3}\left(2 b_{2}+\frac{d_{0}}{d_{2}} r^{2} b_{1}\right),
$$

after correction of a misprint in Eq. (3.17) of ref. $18\left(a+\right.$ sign for the term containing $\left.b_{1}\right)$, with

$$
\left(m_{W}^{*}\right)^{2}=\frac{\beta m_{W}^{2}}{4 \pi \varepsilon_{0} \sigma_{W}^{3}} .
$$

It was verified that the relations derived by $\mathrm{H} \varnothing \mathrm{ye}$ and Lomba ${ }^{50}$ in the restricted case (for $r=1$ ) are recovered from Eqs. (5)-(24). Moreover the latter equations are in keeping with those used by Liu et al. ${ }^{48}$ and by Herzog et al. ${ }^{16}$.

Besides electrostatic interactions, excluded volume between the particles modeled as hard spheres (HS) contributes to the deviations from ideality. For an ion-dipole mixture the excess chemical potential arising from HS volume exclusion was taken from Eq. (30) of previous work ${ }^{51}$, which was found by differentiating the HS Helmholtz energy expression (given on page 3716 of ref. 52) in the Carnahan-Starling approximation. One has,

$$
\beta \mu_{i}^{H S}=-\ln \left(1-X_{3}\right)+\sigma_{i} F_{1}+\sigma_{i}^{2} F_{2}+\sigma_{i}^{3} F_{3}
$$


in which $X_{3}$ is the volume fraction occupied by the particles, and the expressions for $F_{1}, F_{2}$ and $F_{3}$ may be found in ref. 51. For pure solvent $\mathrm{W}$ this equation simplifies to ${ }^{53}$,

$$
\beta \mu_{W}^{H S}=X_{3}\left(8-9 X_{3}+3 X_{3}^{2}\right) /\left(1-X_{3}\right)^{3} .
$$

The pressure of the hard-sphere fluid was expressed by using the equation derived by Boublik $^{54}$ for mixtures,

$$
\beta P^{H S}=\frac{\rho_{t}}{1-X_{3}}+\frac{6}{\pi}\left[\frac{3 X_{1} X_{2}}{\left(1-X_{3}\right)^{2}}+\frac{X_{2}{ }^{3}\left(3-X_{3}\right)}{\left(1-X_{3}\right)^{3}}\right] .
$$

with $X_{n}=(\pi / 6) \sum_{i} \rho_{i} \sigma_{i}{ }^{n}$ and $\rho_{t}=\sum_{i} \rho_{i}$. For pure solvent this expression reduces to the classic Carnahan-Starling formula ${ }^{55}$.

The total excess chemical potential of any species $i$ reads,

and the pressure is given by,

$$
\beta \mu_{i}^{e x c}=\beta \mu_{i}^{H S}+\beta \mu_{i}^{e l} \text {. }
$$

$$
\beta P=\beta P^{H S}+\beta P^{e l} .
$$

\section{B. Gibbs-Duhem equation}

It was verified numerically, by using the symbolic computation program Maple, that the electrostatic contributions to the solute and solvent chemical potentials, and to the pressure (Eqs. (19), (23) and (15), respectively) fulfill the Gibbs-Duhem relation (at constant $T$ ) which may be written as.

$$
-\mathrm{d} P^{e l}+2 \rho_{s} \mathrm{~d} \mu_{i}^{e l}+\rho_{W} \mathrm{~d} \mu_{W}^{e l}=0
$$

in which the factor of 2 is present because every salt 'molecule' releases two ions in water.

Fulfilment of Eq. (30) was examined numerically for various arbitrary values of the concentrations. The differentials $(\mathrm{d}[\cdots])$ were computed in the vicinity of 2 particular concentrations, e.g. $C_{s}=1 \mathrm{~mol} \mathrm{~L}{ }^{-1}$ and $C_{W}=55 \mathrm{~mol} \mathrm{~L}^{-1}$. The Gibbs-Duhem relation was deemed to be verified when the sum of the 3 terms in Eq. (30) was much smaller than any of the 3 terms. This was found to be the case for any values of $C_{s}$ and $C_{W}$, with a typical accuracy of the order of $10^{-7}$ in the Maple program. It was observed that even a slight modification in a single formula of the Maple program resulted in a clear unfulfillment of the Gibbs-Duhem relation. 
This is a new result which suggests, with a high degree of confidence, that all ID-MSA equations given in the preceding section are valid. It also ensures that the numerical values of the pressure and of the chemical potentials (of the salt and of the solvent) are computed correctly in the Maple program.

In the same way it was verified that the HS contributions, $P^{H S}, \mu_{i}^{H S}$ and $\mu_{W}^{H S}$, fulfill the Gibbs-Duhem relation. Lastly the same verification was done for the total salt and solvent chemical potentials (by including the ideal contribution, $\ln \rho_{i}$ with $i=\mathrm{s}$ or $\mathrm{W}$ ) at constant pressure (see Section IV A 2 about how to maintain pressure constant).

\section{Relative permittivity of solution}

Another quantity of interest is Adelman's dieleetric constant ${ }^{56}$ which represents the dielectric constant, or relative permittivity, of solution. It relates to the interaction of two charges at infinite distance in a solution ${ }^{56}$. This latter reference provides a powerful indication of how to compute this quantity in theoretical models of liquids and solutions.

In the present MSA framework, the following expression was obtained ${ }^{18,47}$,

$$
\measuredangle \varepsilon_{A}=1+\frac{d_{2}{ }^{2}}{y_{1}{ }^{2}} \text {. }
$$

by combining Eqs. (1.19), (1.32) and (3.19) of ref. 18. It is noted that this expression yields a concentration-dependent permittivity as observed experimentally ${ }^{33}$.

In the absence of ions, this expression coincides with Wertheim's dielectric constant ${ }^{57}$, $\varepsilon_{W}$, for pure solvent in the MSA. The latter may easily be obtained from the following parametric equations expressed as a function of the polarization parameter $\lambda$ for pure $\mathrm{W}^{19}$,

$$
\begin{gathered}
d_{2}=\frac{\lambda(\lambda+2)}{3} \sqrt{1-\frac{1}{\varepsilon_{W}}}, \\
\varepsilon_{W}=\frac{\lambda^{2}(\lambda+1)^{4}}{16} .
\end{gathered}
$$

D. Mean salt activity coefficient

The chemical potential of an ion may be expressed as,

$$
\beta \mu_{i}=\beta \mu_{i}^{(0), \rho}+\ln \rho_{i}+\beta \mu_{i}^{e x c}
$$


in which $\mu_{i}^{(0), \rho}$ is the standard chemical potential on number density scale (analogous to molar scale), $\ln \rho_{i}$ is the ideal part, and $\mu_{i}^{e x c}$ is the excess chemical potential. In the present framework the latter is composed of two contributions arising from hard core volume repulsion and electrostatic interactions.

Similarly, the activity coefficient of ion $i$ on molal scale, $\gamma_{i}$, is defined by,

$$
\beta \mu_{i}=\beta \mu_{i}^{(0), m}+\ln m_{i}+\ln \gamma_{i}
$$

with $m_{i}$ the molality of the ion and $\mu_{i}^{(0), m}$ its standard chemieal potential on molal scale. For the present system the two monovalent ions have the same chemical potential and activity coefficient (because $\sigma_{+}=\sigma_{-}$), so $\gamma_{+}=\gamma_{-}=\gamma_{s}$, with $\gamma_{s}$ the mean salt activity coefficient.

It is shown in the Appendix that $\gamma_{s}$ may be obtained from the following relation,

$$
\ln \gamma_{s}=\beta \Delta \mu_{i}^{e x c}+\ln \left(\frac{\rho_{W}}{\rho_{W}^{0}}\right) .
$$

where $\Delta \mu_{i}^{e x c}=\beta \mu_{i}^{e x c}-\beta \mu_{i}^{e x c}\left(\rho_{s} \rightarrow 0\right)$, with $\mu_{i}^{e x c}\left(\rho_{s} \rightarrow 0\right)$ the excess chemical potential of an ion at infinite dilution of the salt and $\rho_{W}^{0}$ is the number density of pure solvent.

\section{E. Examination of the chemical potential of the ions at high dilution}

In this section we consider the case of a very dilute solution, for which $d_{0} \ll 1$.

First we focus on the ion-ion contribution $\beta \mu_{i}^{i i}$ (Eq. (20)). At high dilution of the salt, one has (Eq. (3.10b) of ref. 48),

$$
b_{0} \sim-\frac{d_{0}}{2 \sqrt{\varepsilon_{W}}},
$$

Using Eq. (5) and the definition of Debye screening parameter at infinite dilution,

$$
\kappa^{2}=8 \pi L_{0} \rho_{s} / \varepsilon_{W}
$$

one gets in that limit,

$$
d_{0} \simeq \kappa \sigma_{i} \sqrt{\varepsilon_{W}},
$$

Inserting this relation into Eq. (37) and using Eq. (20) one obtains,

$$
\beta \mu_{i}^{i i} \simeq-L_{0} \frac{\kappa}{2} .
$$

This relation reminds one of the Debye-Hückel (DH) limiting law ${ }^{58}$ for the activity coefficient of a salt, except that the r.h.s. should be divided by the dielectric constant $\varepsilon_{W}$. This 
difference is due to the fact that Eq. (40) represents the "true", or "bare Coulomb" 56 , ion-ion contribution corresponding to direct, unshielded, ion-ion interactions. In contrast, the result obtained from primitive (implicit solvent) models, such as DH theory or primitive MSA, includes the effect of the shielding produced by the solvent, which acts as a continuum of relative permittivity $\varepsilon_{W}$.

We now show how the primitive model limiting law may be-recovered in the present framework. At high dilution, the parameter $b_{1}$ may be approximated by a Taylor expansion in powers of $d_{0}$ as,

$$
b_{1} \sim b_{1}^{(1)} d_{0}+b_{1}^{(2)} d_{0}^{2}+\ldots
$$

because $b_{1}=0$ when $\rho_{s}=0$ (salt-free case). The expression of $b_{1}^{(1)}$ has been given elsewhere ${ }^{48}$ (see Supplementary Material). One gets the expression of $b_{1}^{(2)}$ after some simplifications using Eqs. (32), (33) and (39),

$$
b_{1}^{(2)} d_{2}=-\frac{r}{2 \sqrt{\varepsilon_{W}}}\left(1-\frac{1}{\varepsilon_{W}}\right),
$$

Inserting this result into Eq. (41) and using Eq. (39), Eq. (21) may be rewritten,

$$
\beta \mu_{i}^{i d} \sim-\frac{L_{0}}{\sigma_{W}} d_{2} b_{1}^{(1)}+L_{0} \frac{\kappa}{2}\left(1-\frac{1}{\varepsilon_{W}}\right),
$$

Now, by adding the ion-ion and ion-dipole contributions, Eqs. (40) and (43), and after cancelling out the term $L_{0} \kappa / 2$, one gets the expansion of $\beta \mu_{i}^{e l}$ to the first order in $d_{0}$ in the form,

$$
\beta \mu_{i}^{e l} \sim-\frac{L_{0}}{\sigma_{W}} d_{2} b_{1}^{(1)}-\frac{L_{0}}{\varepsilon_{W}} \frac{\kappa}{2}+\ldots,
$$

in which $\kappa$ is proportional to $d_{0}$ by virtue of Eq. 39 .

In this expression, the second term on the r.h.s. is the DH limiting law of the primitive model, which is recovered (as opposed to Eq. (40)). Ionic interactions are now indeed shielded by the dipolar solvent by a factor of $\varepsilon_{W}$. This derivation illustrates the fact that ion-dipole interactions lead to a reduction of the direct ion-ion forces through the effect of the reaction field mediated by the solvent dipoles ${ }^{59}$. This phenomenon is well captured by the ID-MSA model.

The first (constant) term in Eq. (44) is the Gibbs solvation energy for which a more explicit formulation may be derived ${ }^{35,60}$,

$$
\beta \mu_{i}^{\text {Solv }} \sim-\frac{L_{0}}{\sigma_{i}+\sigma_{W} / \lambda}\left(1-\frac{1}{\varepsilon_{W}}\right) .
$$


This expression is similar to the Born equation, but with an extra length in the denominator, $\sigma_{W} / \lambda$, added to the ion diameter (the Born equation is recovered by taking $\sigma_{W}=0$ ). This feature has a clear physical meaning. This added length accounts for the effect of the granularity of the solvent which reduces the solvation energy as compared to the Born model in which the solvent is viewed as a continuum. This interpretation is reminiscent of an old hypothesis made to interpret experimental hydration energies within the Born model ${ }^{61}$.

Eq. (45) has been applied to the description of Gibbs solvation energies in various solvents ${ }^{35,37}$.

\section{F. Ion chemical potential in dilute solutions}

In the previous section it has been seen that in the ID-MSA the ion-ion and ion-dipole contributions to the ion chemical potential are for direct unshielded interactions. However, in classic models for electrolytes, the "Born" term is often added to expressions in which the ion-ion contribution is for shielded electrostatic interactions. So now we would like to derive an expression for ion-dipole interactions from the ID-MSA model, that would be consistent with this feature.

For this purpose it is proposed to first make an effective ion-ion term appear in the expression of the ion chemical potential. This may be done in the case of dilute solutions by isolating such a term in the equation for $\mu_{i}^{e l}$, Eq. (19). Indeed we notice that, following Adelman ${ }^{56}, \varepsilon_{A}$ represents the dielectric constant of solution at large interionic separations. Interactions at such distances contribute predominantly at low concentration.

Consequently, in the limit of dilute solutions in which the correlation functions for the direct ion-ion interactions are divided by $\varepsilon_{A}{ }^{56}$ (thus leading to effective correlation functions), it is proposed to rewrite Eq. (22) in the following different form,

$$
\beta \mu_{i}^{e l}=\beta \mu_{i}^{i i} \frac{1}{\varepsilon_{A}}+\left[\beta \mu_{i}^{i i}\left(1-\frac{1}{\varepsilon_{A}}\right)+\beta \mu_{i}^{i d}\right],
$$

which is equivalent to Eq. (22), but in which the first term represents the effective contribution from shielded ion-ion interactions (direct ion-ion forces reduced by a factor of $\varepsilon_{A}$ ).

$$
\beta \mu_{i}^{i i(e f f)}=\beta \mu_{i}^{i i} \frac{1}{\varepsilon_{A}}
$$

This term gives back the traditional DH mean salt activity coefficient as salt concentration goes to zero as has been shown in the preceding section. 
The second term in brackets in Eq. (46) represents the part of ion-dipole interactions from which the effect of the reaction field on the ions (created by the dipoles) has been subtracted. Hereafter we will focus on this rescaled ion-dipole contribution that is thus defined as,

$$
\beta \mu_{i}^{i d(r e s c)}=\beta \mu_{i}^{i d}+\beta \mu_{i}^{i i}\left(1-\frac{1}{\varepsilon_{A}}\right)
$$

with $\mu_{i}^{i i}, \mu_{i}^{i d}$, and $\varepsilon_{A}$ being expressed by Eqs. (20), (21), and (31), respectively.

From Eqs. (46)-(48) we have the new breakdown of the electrostatic part of the ion chemical potential as,

$$
\beta \mu_{i}^{e l}=\beta \mu_{i}^{i i(e f f)}+\beta \mu_{i}^{i d(\text { resc })}
$$

In what follows we will be interested mainly in the rescaled ion-dipole contribution relative to infinite dilution, $\beta \mu_{i}^{i d(\text { resc })}-\beta \mu_{i}^{\text {Solv }}$. From Eq. 48 one gets,

$$
\beta \Delta \mu_{i}^{i d(r e s c)}=\beta \Delta \mu_{i}^{i d}+\beta \mu_{i}^{i i}\left(1-\frac{1}{\varepsilon_{A}}\right),
$$

in which,

$$
\beta \Delta \mu_{i}^{i d} \equiv \beta \mu_{i}^{i d}-\beta \mu_{i}^{S o l v}
$$

is the direct ion-dipole contribution relative to infinite dilution. Both $\Delta \mu_{i}^{i d}$ and $\Delta \mu_{i}^{i d(\text { resc })}$ tend towards zero when $C_{s} \rightarrow 0$, because then $\beta \mu_{i}^{i i} \rightarrow 0$.

It is noted in Eq. (50) that the last term arising from ion-ion interactions is always negative because ${ }^{19} b_{0}<0$, which makes $\mu_{i}^{i i}<0$ by virtue of Eq. (20), and because $\varepsilon_{A}>1$. It will be seen below that the direct ion-dipole contribution in Eq. (50), $\beta \Delta \mu_{i}^{i d}$, is positive in the ID-MSA model. The rescaled ion-dipole term, $\beta \Delta \mu_{i}^{i d(r e s c)}$, therefore consists of the difference of two (positive) quantities corresponding to the effect of direct ion-dipole and ion-ion interactions.

\section{G. Expansion of the rescaled ion-dipole term at low salt concentration.}

The behavior of this rescaled ion-dipole term (Eq. (50)) may be studied at high dilution by expanding it in powers of $d_{0}$ at constant $d_{2}$, i.e. at constant solvent concentration. The first term in this expansion, in $d_{0}$, is zero because otherwise it would contribute to the ion-ion term (the first term in Eq. (46)). This point was verified by expanding $b_{0}$ and $b_{1}$ in powers of $d_{0}$ and inserting these expansions into Eq. (50). 
The details of the expansion are given in the Supplementary Material addendum. The result is,

$$
\beta \Delta \mu_{i}^{i d(r e s c)} \sim \frac{L_{0}}{\sigma_{i}} M d_{0}^{2}+\mathcal{O}\left(d_{0}^{3}\right)
$$

in which the expression of $M$ is given in Eq. (S18) of Supplementary Material.

It is noticed in this equation that $\beta \Delta \mu_{i}^{i d(\text { resc })}$ is proportional to the salt concentration, $C_{s}$, at low concentration (not to $\sqrt{C_{s}}$ ).

In the expression of $M$ it is observed that, as required, one has $\Delta \mu_{i}^{i d(\text { resc })}=0$ for $\lambda=1$, that is when the ions are placed in a vacuum (one has $\varepsilon_{W}=1$ for $\lambda=1$ according to Eq. $(33))$.

\section{H. "Born" term in ID-MSA framework}

A "Born" term may be proposed in the ID-MSA framework by following the same procedure as for the establishment of Eq. (1). This can be simply done by replacing $\varepsilon_{W}$ by $\varepsilon_{A}$ (given by Eq. (31)) in Eq. (45). Then the equivalent of Eq. (2) in this framework may be obtained by subtracting the solvation energy to this expression, which yields a term in the spirit of the classic "Born" term as,

$$
\beta \Delta \mu_{i}^{B}=\frac{L_{0}}{\sigma_{i}+\sigma_{W} / \lambda}\left(\frac{1}{\varepsilon_{A}}-\frac{1}{\varepsilon_{W}}\right) .
$$

in which $\lambda$ is kept constant vs. salt concentration.

This expression may be expanded in powers of $d_{0}$, again at constant $d_{2}$. The only parameter varying with $d_{0}$ is $\varepsilon_{A}$ which is a function of $y_{1}$ and therefore of $b_{2}$ (cf. Eq. (12)). One obtains,

$$
\beta \Delta \mu_{i}^{B}=\frac{L_{0}}{\sigma_{i}} B d_{0}^{2}+\mathcal{O}\left(d_{0}^{3}\right)
$$

in which the expression of $B$ is given in Eq. (S20).

Eqs. (54) and (S20) show that $\Delta \mu_{i}^{B}$ is always positive (because $\lambda>1$ ), which is also the case in Eq. (53) because $\varepsilon_{A}<\varepsilon_{W}$ as will be seen below. 


\section{RESULTS AND DISCUSSION}

\section{A. Application of ID-MSA model to solvent and ionic solution}

\section{Parameters for pure dipolar solvent}

In what follows we consider the hypothetical dipolar solvent W composed of hard spheres with an embedded point dipole at its center. We want this solvent to have some properties in common with real water. Since the MSA model for an assembly of dipoles involves two parameters (the size and dipole moment of the dipoles), two different properties may be represented exactly. Here it was chosen to describe the density ${ }^{62}$ and the dielectric constant ${ }^{45}$ of water at $1 \mathrm{~atm}$ and $25^{\circ} \mathrm{C}$, that is $d_{W}=0.997047 \mathrm{~kg} \mathrm{dm}^{-3}$ and $\varepsilon_{W}=78.4$.

By solving Eq. (33) with $\varepsilon_{W}=78.4$ one gets $\lambda \simeq 2.65345$. Then by inserting this value for $\lambda$ into the expression for $d_{2}$ (Eq. (32)) one gets $d_{2, W} \simeq 4.0896$ for pure W, and from Eq. (6) one obtains the value of the dipole moment of $\mathrm{W}, m_{W} \simeq 2.2203 \mathrm{D}$. This latter value is larger than for water in the gas phase $(\sim 1.85 \mathrm{D})$, which is satisfactory because polarization effects in liquid water are known to increase the dipole moment of the molecule. Moreover it compares well with values of $2.27 \mathrm{D}$ and $2.35 \mathrm{D}$ in the simple point charge (SPC) and extended SPC models ${ }^{63}$, and reasonably well with a value of $2.6 \mathrm{D}$ for water in ice ${ }^{64}$ (at a time an authoritative result for liquid water) and with values from ab initio numerical simulation ${ }^{65}$ that are in the range of $\sim 2.4 \mathrm{D}^{66}$ to $2.95 \mathrm{D}^{67}$.

The pressure of pure solvent was computed from Eq. (29) in which the Carnahan-Starling formula ${ }^{55}$ and the result of ref. 49 were used for $P^{H S}$ and $P^{e l}$, respectively. The previously determined dipole moment value was inserted into $P^{e l}$. By writing that the total pressure is $1 \mathrm{~atm}$ and by solving this equation with Maple, one gets $\sigma_{W} \simeq 2.4805 \AA$. This value is at the low end of commonly admitted diameters for the water molecule in the literature, that are in the range of $\sim 2.5 \AA$ to $2.9 \AA^{68}$.

\section{Ion-dipole mixture}

Next, the case of an ionic solution made up of dipolar and charged hard spheres at $25^{\circ} \mathrm{C}$ was considered.

There are not many real strong electrolytes that satisfy the condition $\sigma_{+} \simeq \sigma_{-}$. For 
example, if one looks at alkali halides, one finds the following possible candidates. $\mathrm{Na}^{+}$ and $\mathrm{F}^{-}$have close Shannon and Prewitt (SP) diameters ${ }^{43}$ of $2.32 \AA$ and $2.38 \AA$ (for a coordination number of 6), respectively, but $\mathrm{NaF}$ is not a good candidate because this salt exhibits a peculiar association resulting from "localized hydrolysis" ${ }^{69}$, with an association constant of the order of unity ${ }^{70,71}$. Next, one finds $\mathrm{RbCl}$ and $\mathrm{CsBr}$. The SP diameters ${ }^{43}$ of the ions in these salts are $\sigma_{R b^{+}} \sim 3.32 \AA, \sigma_{C l^{-}} \sim 3.34 \AA, \sigma_{C s^{+}} \sim 3.62 \AA$, and $\sigma_{B r^{-}} \sim$ $3.64 \AA$. However, $\mathrm{CsBr}$ also exhibits an association constant of the order of unity ${ }^{72}$. The association constant for $\mathrm{RbCl}$ is smaller $K_{R b C l} \sim 0.26^{73}$ The proportion of pairs, $\alpha_{p}$, in these solutions may be estimated from the relation ${ }^{74} \alpha_{p}=K m_{s} \gamma_{s}{ }^{2} / \gamma_{p}$, by making the approximation $\gamma_{p} \simeq 1$ for the neutral ion pair at low concentration. The result from this estimation was confirmed by the use of the prinitive MSA model ${ }^{75}$, indicating that a 0.5 mol $\mathrm{kg}^{-1} \mathrm{RbCl}$ solution would contain $\sim 5 \%$ of ion pairs, while the proportion would be $20 \%$ in a similar $\mathrm{CsBr}$ solution. Therefore $\mathrm{RbCl}$ seems to be the best candidate here. It will be chosen preferentially in the applications below.

In practice the ID-MSA equations were solved as follows. For given values of $d_{0}$ and $d_{2}$, Eqs. 7-9, and the equation for the pressure if the latter is maintained constant, were solved numerically by using the symbolic calculus software Maple. The pressure was maintained constant by determining numerically, for each salt concentration, the concentration of water molecules that gave a pressure of $1 \mathrm{~atm}$ for the ion-dipole mixture. Since the ID-MSA equations admit several solutions for $b_{0}, b_{1}$ and $b_{2}$, an approximate guess is useful to obtain the unique physical solution. Here, approximate values based on the formulas proposed by Harvey $^{76}$ were used as initial input in Maple for the lowest concentration. Then the salt concentration was gradually and slowly incremented up to a typical maximum concentration of $1 \mathrm{M}$. Conveniently, the solution found for a given concentration was used as a set of initial values for the next higher concentration.

The mean salt activity coefficient on molal scale was obtained from Eq. (36). The molality of the salt in the solution was calculated from the formula,

$$
m_{s}=\frac{1}{M_{w}} \frac{\rho_{s}}{\rho_{W}}
$$

in which $M_{w}$ is the molar mass of water. 


\section{Some results in the case of a model solution of $\mathrm{RbCl}$ (and $\mathrm{CsBr}$ and $\mathrm{NaF}$ )}

We are going to consider mainly a model solution built up of $\mathrm{Rb}^{+}$and $\mathrm{Cl}^{-}$ions dissolved in the dipolar solvent W characterized in Section IV A 1. It is interesting to look at the results for a few physical properties obtained from the ID-MSA model. Here we calculated at constant pressure: the Gibbs solvation energy, the mean salt activity coefficient, the specific volume, and the relative solution permittivity. Their magnitude was compared with experimental data.

Experimental data and results from the ID-MSA model for the Gibbs hydration energies of $\mathrm{Rb}^{+}$and $\mathrm{Cl}^{-}$ions are given in Table I. As was done by Fawcett ${ }^{37}$, we neglect the dipoledipole contribution ${ }^{36}$ to the theoretical solvation energy. Then, $\beta \mu_{i}^{\text {Solv }}$ represents the Gibbs hydration energy of the ion. Values for a few other ions are also collected in the table. They will be useful for discussion below. Experimental data were taken from papers by Fawcett ${ }^{37}$ and Kelly ${ }^{77}$.

TABLE I. Gibbs hydration energies of ions (in units of $k_{B} T$ ).

\begin{tabular}{|c|c|c|c|c|c|}
\hline Ion & $\sigma_{i}^{a}(/ \AA$ & & xp. data ${ }^{c}$ & ID-MSA result & Deviations $^{d}$ \\
\hline $\mathrm{Na}^{+}$ & & & -174.0 & -170.0 & $0.6 \% / 2.3 \%$ \\
\hline $\mathrm{Rb}^{+}$ & & & -135.9 & -130.0 & $2.3 \% / 4.3 \%$ \\
\hline & & & -126.6 & -121.5 & $1.2 \% / 4 \%$ \\
\hline & & -173 & -176.0 & -166.9 & $3.5 \% / 5.2 \%$ \\
\hline & 3.34 & -123 & -125.6 & -129.4 & $-5.2 \% /-3 \%$ \\
\hline & 264 & -112 & -115.2 & -120.9 & $-7.9 \% /-4.9 \%$ \\
\hline
\end{tabular}

${ }^{a}$ From ref. $43 ;{ }^{b}$ From Fawcett ${ }^{37} ;{ }^{c}$ From Kelly ${ }^{77} ;{ }^{d}$ Relative deviation of ID-MSA result w.r.t. Fawcett/Kelly data.

It is seen in this table that the ID-MSA result is smaller (in absolute value) than the experimental data in the case of $\mathrm{Rb}^{+}$, and it is larger in the case of $\mathrm{Cl}^{-}$. The average IDMSA value is -129.7. The average value of $\beta \mu_{i}^{\text {Solv }}$ obtained from the model is just between, and in good agreement with, the experimental mean ionic Gibbs hydration energies of -128 (from Fawcett) and -130.8 (from Kelly). It is besides noticed in the table that the data of Fawcett and Kelly exhibit a nearly constant discrepancy of $\sim 3$, due to different absolute 
values adopted for the proton $\left(\beta \mu_{i}^{\text {Solv }}=-445.6\right.$ and -448.4 , respectively $)$. The theoretical results for the cations are in better agreement with experimental data than those for the anions. The largest deviation is found in the case of $\mathrm{Br}^{-}$.

Experimental values for the mean salt activity coefficient on molal scale, $\gamma_{s}$, were retrieved from a famous compilation of recommended values ${ }^{42}$. The result from the ID-MSA model obtained from Eq. (36) with the common ion diameter of $3.33 \AA$ (average SP value) is plotted in Figure 3 together with experimental data for molalities up to 1 mol $\mathrm{kg}^{-1}$. The sensitivity of the activity coefficient to the value of the common ion diameter is shown by also plotting the results for $\sigma_{i}=3.23 \AA$ and $3.43 \AA$. The calculated $\gamma_{s}$ for $\sigma_{i}=3.33 \AA$ is in

FIG. 3. Activity coefficient (on molal scale) for $\mathrm{RbCl}$ at $25^{\circ} \mathrm{C}$. Symbols $=$ experimental values; Solid line $=$ result from the model for $\sigma_{i}=3.33 \AA$; Bottom dashed curve = result for $\sigma_{i}=3.23 \AA$; Top dashed curve $=$ result for $\sigma_{i}=3.43 \AA$.

excellent agreement with the experimental data for $m \leq 0.4 \mathrm{~mol} \mathrm{~kg}^{-1}$. This is not so for the other two $\sigma_{i}=$ values. At higher concentrations, the plot for $\gamma_{s}$ deviates progressively from the experimental points. At $1 \mathrm{~mol} \mathrm{~kg}^{-1}$ the latter are closer to the curve obtained for $\sigma_{i}=3.43 \AA$.

It is worth breaking down the various contributions to $\ln \gamma_{s}$. By virtue of Eqs. (22), (28), 
(36), and (49), one has,

$$
\begin{aligned}
\ln \gamma_{s} & =\beta \mu_{i}^{i i}+\beta \Delta \mu_{i}^{i d}+\beta \Delta \mu_{i}^{H S}+\ln \left(\frac{\rho_{W}}{\rho_{W}^{0}}\right) \\
& =\beta \mu_{i}^{i i(e f f)}+\beta \Delta \mu_{i}^{i d(r e s c)}+\beta \Delta \mu_{i}^{H S}+\ln \left(\frac{\rho_{W}}{\rho_{W}^{0}}\right) .
\end{aligned}
$$

It was found that the effective ion-ion contribution, $\beta \mu_{i}^{i i(e f f)}(\mathrm{Eq}$ (47)), is much larger than the rescaled ion-dipole contribution, $\beta \Delta \mu_{i}^{i d(\text { resc })}$ (Eq. (50)), below 1M. The ratio of the two is $\sim-50$ at $0.1 \mathrm{M}$ and $\sim-25$ at $1 \mathrm{M}$. The HS contribution is $\sim 6$ times and $\sim 2$ times smaller than the ion-ion contribution at $0.1 \mathrm{M}$ and $1 \mathrm{M}$, respectively. The contribution corresponding to the term $\ln \left(\rho_{W} / \rho_{W}^{0}\right)$ in Eq. 36 is of the same order as the rescaled ion-dipole contribution.

It is also worth mentioning at this point that, as is common with this type of explicitsolvent model, the direct ion-ion, $\beta \mu_{i}^{i i}$ (Eq. 20), and ion-dipole, $\beta \Delta \mu_{i}^{i d}$ (Eq. 51), contributions for unshielded interactions vary much more abruptly, and are of much larger magnitude, than $\beta \mu_{i}^{i i(e f f)}$ and $\beta \mu_{i}^{i d(r e s c)}$ do. For example, in the case of $\mathrm{RbCl}\left(\sigma_{i}=3.33 \AA\right)$, the values of these various contributions are collected in Table II at $0.1 \mathrm{M}$ and $0.4 \mathrm{M}$ (notice that in each case, $\left.\beta \mu_{i}^{i i}+\beta \Delta \mu_{i}^{i d}=\beta \mu_{i}^{i i(e f f)}+\beta \Delta \mu_{i}^{i d(\text { resc })}\right)$.

TABLE II. Contributions to the chemical potential of an ion.

\begin{tabular}{ccccc}
\hline$C_{s}$ & $\beta \mu_{i}^{i i}$ & $\beta \Delta \mu_{i}^{i d}$ & $\beta \mu_{i}^{i i(e f f)}$ & $\beta \Delta \mu_{i}^{i d(\text { resc })}$ \\
\hline $0.1 \mathrm{M}-23.9906$ & 23.6741 & -0.3233 & 0.0069 \\
$0.4 \mathrm{M}$ & -42.5239 & 41.8898 & -0.6514 & 0.0173 \\
\hline
\end{tabular}

It should be emphasized that the direct ion-dipole contributions, $\beta \Delta \mu_{i}^{i d}$ (Eq. 51), was found to be positive in all cases. This may be explained by the fact that, when ions arrive in the vicinity of a previously isolated ion X (at infinite dilution), these ions may orientate most of the dipoles around $\mathrm{X}$ in a less favorable manner. Moreover the arrival of ions in the vicinity of $\mathrm{X}$ removes dipoles (those that were present in the volume now occupied by the ions). These phenomena reduce the attractive interaction energy of the central ion $\mathrm{X}$ with the surrounding dipoles.

The activity coefficient $\gamma_{s}$ was also computed in the case of CsBr by taking the average SP diameter of the two ions, $\sigma_{i}=3.63 \AA$. The result is above experimental data below 0.1 mol kg ${ }^{-1}$ (see Figure S4 in Supplementary Material). The deviation is $\sim 5 \%$ at $0.1 \mathrm{~mol}$ $\mathrm{kg}^{-1}$ at which the expected proportion of pairs is $\sim 6 \%$. The discrepancy observed for $\gamma_{s}$ 
may be attributable to association in this solution (the inclusion of association would lower the theoretical value of $\left.\gamma_{s}\right)$. In the case of $\operatorname{NaF}\left(\sigma_{i}=2.35 \AA\right)$, the theoretical $\gamma_{s}$ is located much below the experimental points (see Figure S5) although this salt is deemed to be associated. In this respect NaF exhibits an unexpected behavior within the ID-MSA model, which is quite different than that of $\mathrm{RbCl}$ and $\mathrm{CsBr}$. This behavior may be explained by the occurrence of "localized hydrolysis" involving the fluoride ion which may produce a very special type of ion association ${ }^{69}$. This confirms that this electrolyte is not a good candidate for the present study. Unfortunately no other salt could be identified that is composed of small ions of equal size.

Next, the specific volume (i.e. the volume of solution per $\mathrm{kg}$ of solvent), $V$, was computed using the relation, $V=m_{s} / C_{s}$, in which $m_{s}$ is derived from Eq. (55). The experimental value of $V$ was obtained using the formula, $V=\left(1+m_{s} M_{s}\right) / d$, with $M_{s}$ the molar mass and $d$ the solution density. The densities of $\mathrm{RbCl}$ solutions were computed by utilizing a simple parametrization ${ }^{46}$. The results at constant pressure are plotted in Figure 4 for the average $\mathrm{Rb}^{+}-\mathrm{Cl}^{-}$diameter $\sigma_{i}=3.33 \AA$, and for $\sigma_{i}=3.33 \pm 0.1 \AA$, together with the experimental values. It is seen that the result for $\sigma_{i}=3.33 \AA$ is very much in keeping with the experimental data up to 4 mol $\mathrm{kg}^{-1}$. Thus the model provides a good description of the volumetric properties of $\mathrm{RbCl}$ solutions in standard conditions.

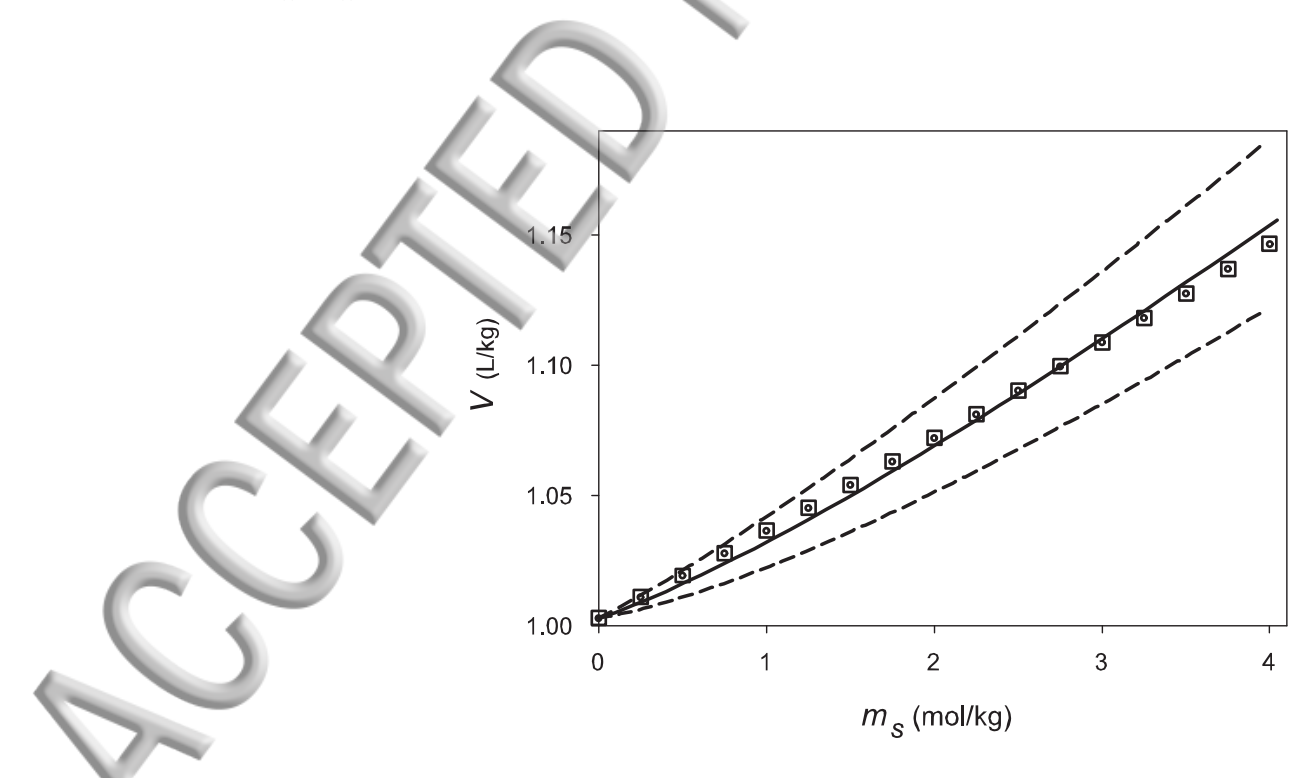

FIG. 4. Specific volume, $V$, of $\mathrm{RbCl}$ solution as a function of salt concentration. $(\square)=$ experimental data; Solid line $=$ ID-MSA result for average $\mathrm{Rb}^{+}-\mathrm{Cl}^{-}$SP diameter of $3.33 \AA$; Bottom dashed line $=$ ID-MSA result for $\sigma_{i}=3.23 \AA$; Upper dashed line $=$ ID-MSA result for $\sigma_{i}=3.43 \AA$. 
These results show that, despite the simple representation of the water molecule, the ID-MSA model gives values for $\beta \mu_{i}^{\text {Solv }}, \gamma_{s}$ and $V$, that are in overall good agreement with experimental data for $\mathrm{RbCl}$ aqueous solutions. Furthermore no adjustable parameter has been introduced for the ions. The description only includes two parameters for the solvent (see Section IV A 1).

Lastly, the relative permittivity of the solution derived from the MSA model, $\varepsilon_{A}$ given by Eq. (31), was calculated as a function of salt concentration. It is plotted in Figure 5 together with raw experimental data ${ }^{33,44}$. It is observed that the calculated $\varepsilon_{A}$ is weakly dependent on the value of $\sigma_{i}$ in the range of $3.23 \AA$ to $3.43 \AA$ (dashed lines), and that it deviates greatly from the experimental data for $\mathrm{RbCl}$ solution.

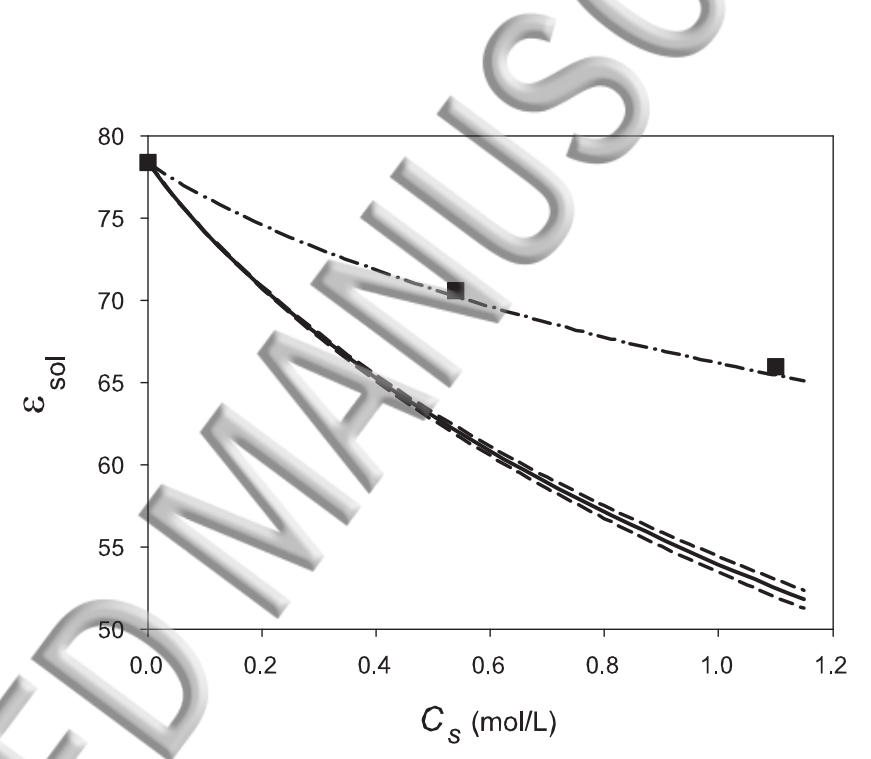

FIG. 5. Relative permittivity of model RbCl solution as a function of salt concentration: $(\mathbf{\square})=$ experimental data; Solid line $=$ ID-MSA value of $\varepsilon_{A}$ for $\sigma_{i}=3.33 \AA$; Upper dashed line $=$ idem for $\sigma_{i}=3.23 \AA$; Bottom dashed line $=$ idem for $\sigma_{i}=3.43 \AA$; Top dash-dotted line $=$ corrected value $\varepsilon_{A}^{\prime}$ (see Eq. (57)) for $\sigma_{i}=3.33 \AA$.

It is likely that this discrepancy originates from the fact that in the ID-MSA model the cation and the anion are solvated in a similar way by the solvent dipoles. Indeed this is not the case in real solutions in which the chloride anion interacts with water through very short-lived hydrogen bonds ${ }^{78,79}$, with the consequence that this ion is very weakly hydrated in all solutions ${ }^{78}$. It is noted that this feature does not have a detectable influence on the other properties studied above. 
In passing, it may be noticed that this fact can be taken into account at sufficiently low concentration by introducing in a semi-empirical way a 'corrected' $\varepsilon_{A}^{\prime}$ as,

$$
\varepsilon_{A}^{\prime} \equiv \frac{\varepsilon_{A}+78.4}{2} .
$$

by which it is assumed that the chloride anion does not modify the solution permittivity (if the effect of the volume occupied by $\mathrm{Cl}^{-}$is neglected). This equation means that only one half of the variation of $\varepsilon_{A}$ (corresponding to the cation) is taken into account in $\varepsilon_{A}^{\prime}$, that is $78.4-\varepsilon_{A}^{\prime}=\left(78.4-\varepsilon_{A}\right) / 2$, which gives Eq. (57). It is seen in Figure 5 that $\varepsilon_{A}^{\prime}$ is in very good accord with raw experimental data.

\section{B. Comparison of "Born" term and rescaled ion-dipole contribution}

Since, as seen in the previous section, the cation and the anion are similarly solvated by the purely dipolar solvent, it stems that the analysis below is not supposed to be applicable to real aqueous solutions in a quantitative manner. Instead, the results for ionic solutions in the dipolar solvent $\mathrm{W}$ will be discussed within the theoretical framework of the ID-MSA model. Nevertheless, the realistic results obtained in the previous section for various physical properties suggest that the results of this section may have some relevance in the case of real dilute aqueous solutions.

The values of the model parameters for $\mathrm{W}$, namely $m_{W}$ or $\lambda$, and $\sigma_{W}$, determined in Section IV A 1 were utilized to study the ion-dipole contribution in model $\mathrm{RbCl}$ solutions at constant solvent concentration (constant $d_{2}$ ) and at constant pressure.

The magnitude of the "Born" term (Eq. (53)) was compared with that of the rescaled ion-dipole term (Eq. (50)).

First the comparison was made in the case of $\mathrm{RbCl}\left(\sigma_{i}=3.33 \AA\right)$ for sufficiently low concentrations, so that the conclusions of Section III F, and Eq. (48) in particular, may be

valid. The results for $\beta \Delta \mu_{i}^{i d(r e s c)}$ (Eq. (50)) and $\beta \Delta \mu_{i}^{B}$ (Eq. (53)), for an ion $i=+$ or -, are plotted in Figure 6 up to $1 \mathrm{M}$. It is observed that the result from the ID-MSA model is much smaller than the two different "Born" terms (dashed line and symbol), which are coincidentally close to each other.

The same quantities are plotted in Figure 7 for $\sigma_{i}=3 \AA$. It is seen that in this case the ID-MSA result is of negative sign whilst the "Born" term is always positive. 


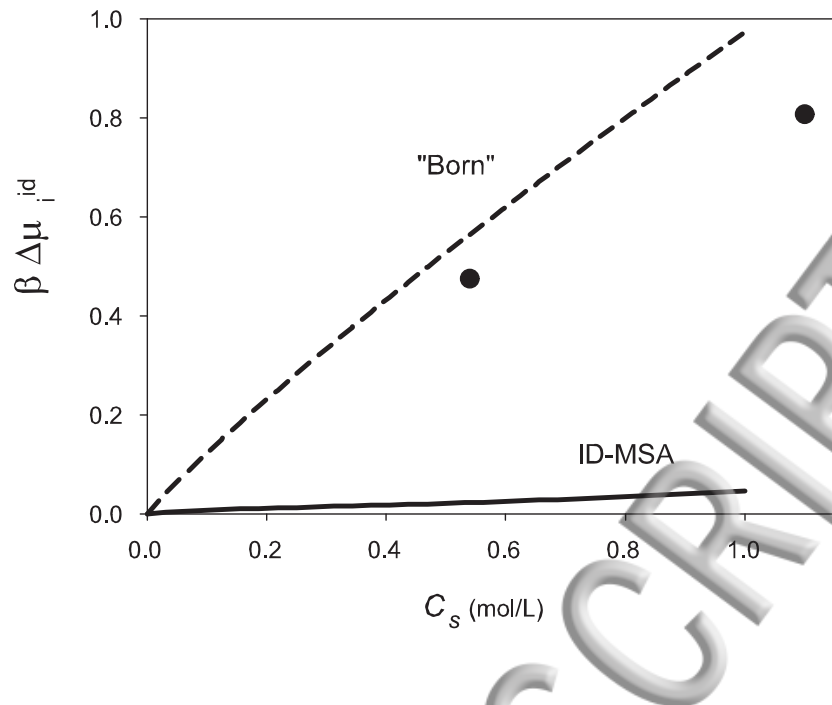

FIG. 6. Rescaled ion-dipole contribution to the ion chemical potential, $\beta \Delta \mu_{i}^{i d(r e s c)}$ (Eq. (50), solid line) and "Born" term (Eq. (53), dashed line) at constant pressure as a function of salt concentration, in the case of $\operatorname{RbCl}\left(\sigma_{i}=3.33 \AA\right)$; Symbol $(\bullet)=$ result for "Born" term in the case of RbCl obtained from Eqs. (2) and (4) (see Figure-S3).

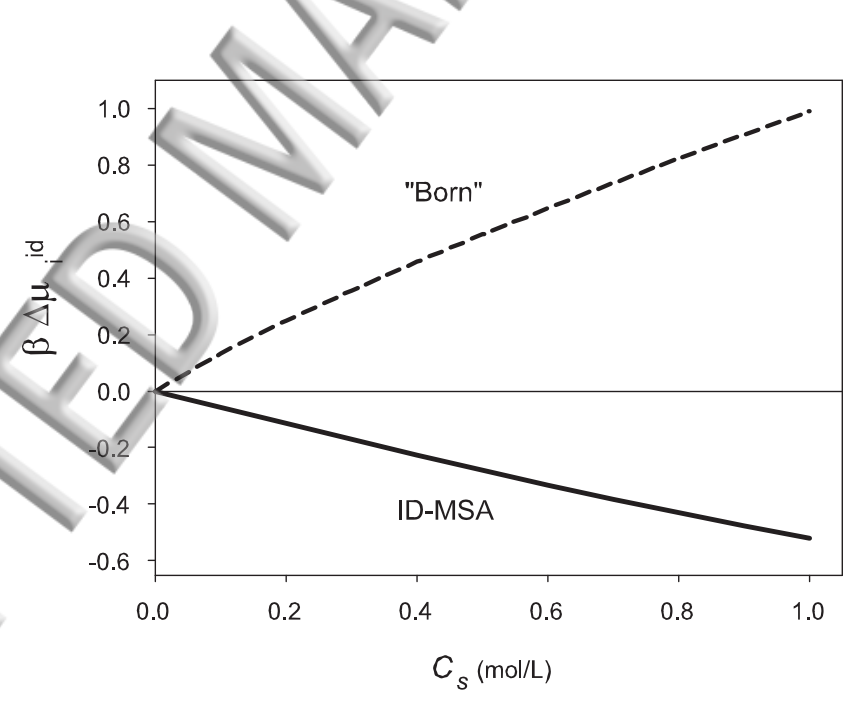

FIG. 7. Same legend as for Figure 6 in the case $\sigma_{i}=3 \AA$.

It will be convenient to define the ratio,

$$
R \equiv \lim _{C_{s} \rightarrow 0}\left(\beta \Delta \mu_{i}^{i d(\text { resc })} / \beta \Delta \mu_{i}^{B}\right)
$$

at vanishing concentration of the salt. It is a function of the sole diameter $\sigma_{i}$ for a given solvent characterized by the values of $m_{W}$ and $\sigma_{W}$. This ratio can be computed at constant 
solvent concentration, in which case, according to the discussion of Section III F, $R$ is expected to be an exact result within the ID-MSA model. It can also be computed numerically at constant pressure in a Maple program.

The ratio $R$ was first computed at constant solvent concentration (constant $d_{2}$ ) for which the two chemical potentials are respectively given by Eqs. (52) and (54). Thus in this case one has, $R=M / B$, in which $M$ and $B$ are expressed by Eqs. (S18) and (S20) (cf. Supplementary Material), respectively. By using the expressions of $M$ and $B$, and the values of $\lambda$ and $\sigma_{W}$ of Section IV A 1 one gets,

$$
R \simeq \frac{0.75154\left(\sigma_{i}+4.6138\right)\left(\sigma_{i}-3.5364\right)}{\left(\sigma_{i}+0.93482\right)\left(\sigma_{i}+0.63715\right)}
$$

in which $\sigma_{i}$ is expressed in Angströms.

Next, the ratio $R$ was computed at constant pressure for $\sigma_{i}$ values ranging from $2.3 \AA$ to $4.5 \AA$ thus spanning the range of ions from fluoride $(2.38 \AA)$ to iodide $(4.12 \AA)$ and beyond. Since an analytical determination of this ratio turned out to be extremely cumbersome, it was computed manually within the Maple program by calculating its value numerically for vanishingly small values of the salt concentration.

The ratio $R$ is plotted in Figure 8 as a function of the ion diameter in the two cases: constant $d_{2}$ (see Eq. (59)), and constant $P$.

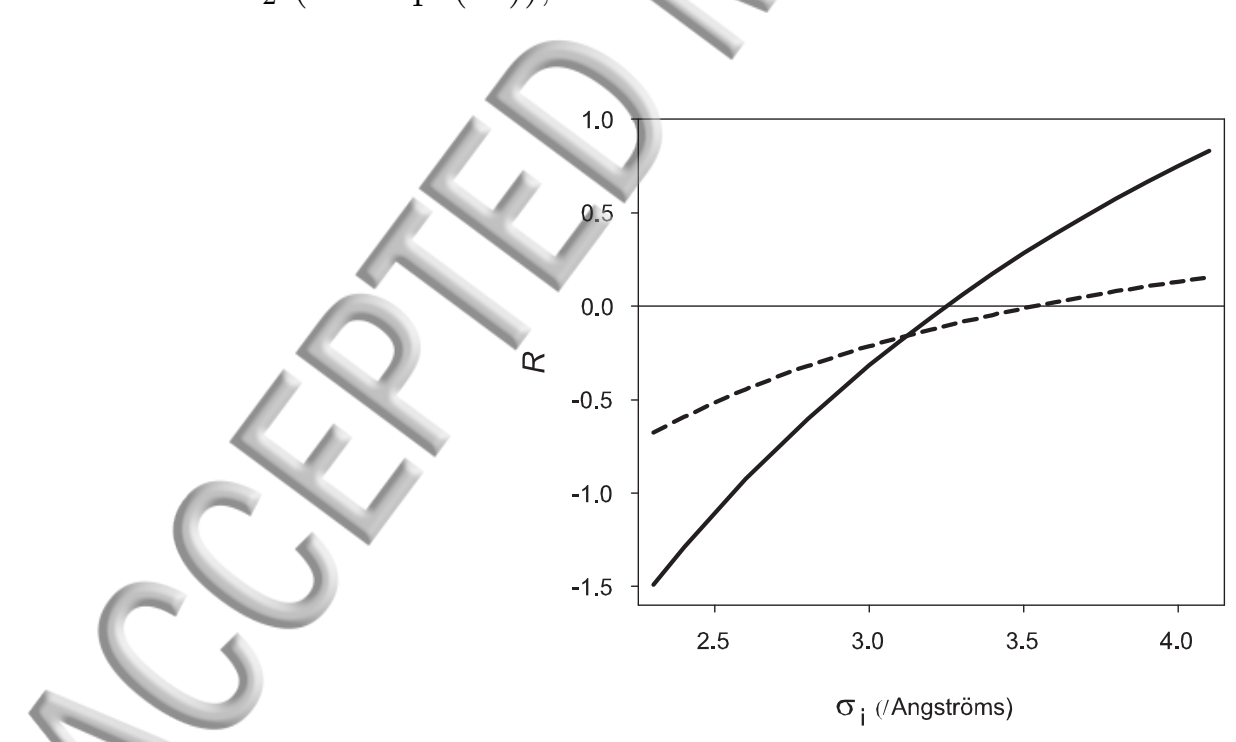

FIG. 8. Plot of $R$ (Eq. (58)) as a function of the ion diameter. Solid line: result at constant pressure; Dashed line: result at constant solvent concentration (Eq. (59)).

The first conclusion that can be drawn from Figures (6)-(8) is that, in general, the result 
for the rescaled ion-dipole contribution to the chemical potential of an ion, calculated from the ID-MSA model, is not well estimated by the "Born" term. In the case of RbCl (average SP diameter, $\sigma_{i}=3.33 \AA$ ) at constant $P$, Figures 6 and 8 show that the MSA result is of the order of 20 times smaller than the "Born" term, which is quite a large number.

Furthermore, Figures 7 and 8 suggest that the ratio $R$ could be negative. In this case $\beta \Delta \mu_{i}^{i d(r e s c)}$ would be negative because the "Born" term being always positive. According to Figure 8 this would occur for $\sigma_{i} \lesssim 3.25 \AA$ at constant pressure, and for $\sigma_{i} \lesssim 3.54 \AA$ at constant solvent concentration (see Eq. (59)). If NaF was not subject to hydrolysis, an aqueous solution of this salt, for which the mean ion diameter is $\sigma_{\bar{i}}=2.35 \AA$, would fall into this category, with an ion-solvent contribution that would be negative and $\sim 1.4$ times the magnitude of the "Born" term in absolute value

At the opposite, it is seen in Figure 8 that at constant pressure one gets $R=1$ for ion diameters of the order of $4.3 \AA$. For this particular diameter the contribution estimated from the model coincides with the "Born" term. For bigger ions $R$ is found to increase beyond unity.

The fact that the ion-dipole contribution, $\beta \Delta \mu_{i}^{i d(\text { resc })}$, differs in general from the "Born" term should not come as a surprise. The "Born" term is based on the view that the medium around an ion is a dielectric of relative permittivity $\varepsilon_{s o l}$. However, $\varepsilon_{\text {sol }}$ is relevant for the description of interactions between charges separated by a sufficiently large distance. It will have a sound and unique physical meaning when this separation is appreciably larger than the mean distance between ions. This is typically a matter of several nanometers in solution. One finds that this minimum-+ distance would be of as much as $\sim 100 \AA$ in a $0.1 \mathrm{M}$ solution, and of $\sim 30 \AA$ in a $6 \mathrm{M}$ solution.

In contrast, the "Born" term is actually intended to quantify the effect of electrostatic ionsolvent interactions. Yet, the range of these interactions (which may include ion-dipole, ionquadrupole,... interactions) is shorter than that of ion-ion interactions. It mainly involves distances of a few molecular diameters. At such distances, $\varepsilon_{\text {sol }}$ is likely a poor estimate of the effective local relative permittivity. This phenomenon might be a basic drawback of the "Born" term.

We note that this mismatch does not prevail in the calculation of ion solvation energies when using the Born equation because the dielectric constant of the solvent, $\varepsilon_{W}$, should be physically relevant already at distances of a few molecular diameters. 
Now, a precise discussion of the variation of $R$ with $\sigma_{i}$ is difficult, firstly because the "Born" term does not have a sound physical basis. Moreover, the magnitude of the rescaled ion-dipole contribution, $\Delta \mu_{i}^{i d(r e s c)}$, is also difficult to discuss because, as mentioned in Section IIIF, it represents the part of direct ion-dipole interactions from which the effect of the reaction field on the ions (created by the dipoles) has been subtracted. It has been seen above (at the end of Section IIIF and in Section IV A 3) that the direct ion-dipole contribution, $\Delta \mu_{i}^{i d}$, is positive, and the ion-ion contribution, $\mu_{i}^{i i}$, is negative. Consequently, by virtue of Eq. (50), $\beta \Delta \mu_{i}^{i d(r e s c)}$ is the difference of two positive quantities that are significantly larger than $\beta \Delta \mu_{i}^{i d(\text { resc })}$, as has been mentioned in Section IV A 3. Therefore, the uncertainty on $\beta \Delta \mu_{i}^{i d(r e s c)}$ may not be small.

Nonetheless the result in the case of RbCl may be trustable. Indeed, it has been found in Section IV A 3 that the solvation energy of $\mathrm{Rb}^{+}$and $\mathrm{Cl}^{-}$ions is well described within the ID-MSA model. Moreover, the activity coefficient of $\mathrm{RbCl}$, which comprises contributions from ion-ion and ion-dipole interactions, is represented accurately below 0.4 M (see Figure 3 ) without adjustable parameter for the ions. These facts may lend support to the result of Figure 6 in which it is found that the rescaled contribution $\beta \Delta \mu_{i}^{i d(r e s c)}$ is much smaller that the "Born" term in the case of RbGl solutions.

The case of the salt $\mathrm{CsBr}$ (made up of ions that are bigger than $\mathrm{Rb}^{+}$and $\mathrm{Cl}^{-}$), may be examined likewise. The mean Gibbs hydration energy of $\mathrm{Cs}^{+}$and $\mathrm{Br}^{-}$obtained from the model is -121.2, compared to the experimental values of $\sim-118$ and $\sim-121$ from Fawcett and Kelly, respectively. The agreement is therefore very good. The activity coefficient of $\mathrm{CsBr}$ is in reasonable agreement with experimental data if one takes into account the fact that this salt is associated in water (see Section IV A 3 and Figure S4). These outcomes may lend some support to the result of Figure 8 in the case of the bigger ions.

In contrast, the case of smaller ions $\left(\sigma_{i} \lesssim 3.25 \AA\right.$ ), for which $\beta \Delta \mu_{i}^{i d(r e s c)}$ would be negative, is more uncertain. It must be admitted that this result is somewhat surprising because it would mean that the rescaled ion-dipole contribution could be of opposite sign to the direct one. Admittedly, the ID-MSA gives reasonable values for the Gibbs hydration energies of $\mathrm{Na}^{+}$and $\mathrm{F}^{-}$(see Table I), but the activity coefficient is not well represented within the IDMSA (see Figure S5), probably because of the occurrence of hydrolysis in NaF solutions as mentioned in Section IV A 3. These results therefore do not provide support to the validity of the model in the case of small ions. For the time being we can just take note of the result 
provided by the ID-MSA that $\Delta \mu_{i}^{i d(\text { resc })}$ might be negative in this case, but this result would have to be confirmed by other methods.

\section{CONCLUSION}

The main conclusions stemming from the above analysis are that the solution permittivity, which is a basic ingredient of the "Born" term, is likely not relevant for the description of ion-solvent effects in electrolyte solutions. Moreover, it has been found within the semirestricted ID-MSA framework that, in general, the "Born" term does not provide a good estimate of the magnitude of the ion-solvent contribution to the chemical potential of an ion in solution.

It has been mentioned in the Supplementary Material addendum that, in the literature, electrolyte models that include a "Born" term have not used experimental solution permittivity data. Instead, the use of approximate estimates gives $\varepsilon_{\text {sol }}$ values that are significantly larger than raw experimental data at $25^{\circ} \mathrm{C}$ (see Figure S2). As a consequence the "Born" contribution is appreciably smaller (see Figure S3). The use of such a smaller "Born" term to describe the ion-dipole contribution coincides with the fact that the "Born" term greatly overestimates the ion-dipole contribution for most systems (see Figures 6 and 8).

It has also been found in this work that the ion-dipole contribution, $\beta \mu_{i}^{i d(r e s c)}$, can be negative when the ions are sufficiently small. If this would indeed occur, then no term of the Born-type could account for this phenomenon because the "Born" term is always positive. However it has been mentioned that this result is not clearly established.

The potential inadequacies of the "Born" term to give a good estimate of the ion-solvent contribution are cause for concern. If the "Born" term is wrong by a few $k_{B} T$ (especially if the actual ion-solvent term is negative) when it is used in a model for electrolyte solutions, then the other parameters of the model have to compensate for this inaccuracy, which may lead to parameters having unphysical values. Against this background, we suggest that more flexibility should be allowed for the "Born" term in electrolyte models. It may be observed in Figures 6 and 7 that the ratio of the ID-MSA contribution to the "Born" term does not vary much with the salt concentration. Consequently, a first modification could be to introduce an adjustable parameter in the "Born" term, such as a prefactor whose value would expectedly fall in the range of -2 to 2 . 
The ID-MSA model offers a convenient possibility to study this topic, but it is clearly an approximate theory. So, the present conclusions would need to be confirmed by another method. Nonetheless there are not many theoretical routes to tackle this problem. Numerical simulation (e.g., molecular dynamics) will likely be the only alternative to study this subject.

Besides this project, it will be attempted in subsequent work to derive an approximate analytical formula for the ion-dipole contribution. It will also be-attempted to develop the same kind of study in the framework of the unrestricted ID-MSA model in which the cation and the anion have different sizes. However this will require first to revisit the model in detail. It will also be interesting to study the results yielded by this model for the deviations from ideality in electrolyte solutions, which has not been done yet.

\section{Appendix}

By equating Eqs. 34 and 35 and taking the limit of infinite dilution of the salt one gets,

$$
\beta \mu_{i}^{(0), \rho}-\beta \mu_{i}^{(0), m}=-\ln \left(\frac{\mathcal{N}}{V_{0}}\right)-\beta \mu_{i}^{e x c}\left(\rho_{s} \rightarrow 0\right),
$$

in which $\mathcal{N}$ is Avogadro's number and $V_{0}$ is the specific volume of the solvent. Then, using Eqs. 34 and 35 for finite salt concentration, one finds,

$$
\ln \gamma_{i}=\ln \gamma_{s}=\beta\left[\mu_{i}^{e x c}-\mu_{i}^{e x c}\left(\rho_{s} \rightarrow 0\right)\right]+\ln \left(\frac{V_{0}}{V}\right) .
$$

with $V$ is the specific volume of solution, viz. the volume of solution per kilogram of solvent. Now one has that, $\rho_{W}=N_{W}^{(1)} / V$, in which $N_{W}^{(1)}$ is the number of solvent molecules in $1 \mathrm{~kg}$ of solvent. Therefore, $V_{0} / V=\rho_{W} / \rho_{W}^{0}$, which together with Eq. (A.2) yields Eq. (36). 


\section{SUPPLEMENTARY MATERIAL}

See supplementary material for more details on the "Born" term in the literature, on the expansion of rescaled ion-dipole and "Born" contributions at low salt concentration, and on the activity coefficients of $\mathrm{CsBr}$ and $\mathrm{NaF}$ in solution.

\section{ACKNOWLEDGMENTS}

The author acknowledges fruitful discussions with colleagues at LFPEN (J.C. de Hemptinne, N. Ferrando, S. Ahmed) and with O. Bernard (Laboratoire PHENIX) about the "Born" term, and with the late L. Blum about the ion-dipole MSA model.

\section{REFERENCES}

${ }^{1}$ H. Renon, Fluid Phase Equilib. 30, 181(1986).

${ }^{2}$ J. M. Prausnitz, R. N. Lichtenthaler, and E. G. de Azevedo, Molecular thermodynamics of fluid-phase equilibria (Prentice Hall, 1999).

${ }^{3}$ J.-L. Cruz and H. Renon, AIChE J. 24, 817 (1978).

${ }^{4}$ C.-C. Chen, H. I. Britt, J. Boston, and L. Evans, AIChE J. 28, 588 (1982).

${ }^{5}$ B. Sander, A. Fredenslund, and P. Rasmussen, Chem. Eng. Sci. 41, 1171 (1986).

${ }^{6}$ W. Chapman, K. Gubbins, G. Jackson, and M. Radosz, Fluid Phase Equilib. 52, 31 (1989).

${ }^{7}$ M. Wertheim, J. Chem. Phys. 87, 7323 (1987).

${ }^{8}$ J. A. Myers, S. I. Sandler, and R. H. Wood, Ind. Eng. Chem. Res. 41, 3282 (2002).

${ }^{9}$ L. Blum, in/Theoretical Chemistry, Advances and Perspectives, Vol. 5, edited by H. Eyring and D. Henderson (Academic Press: New York, 1980) pp. 1-66.

${ }^{10}$ B.-S. Lee and K.-C. Kim, Korean J. Chem. Eng. 26, 1733 (2009).

${ }^{11}$ J. M. Schreckenberg, S. Dufal, A. J. Haslam, C. S. Adjiman, G. Jackson, and A. Galindo, Mol. Phys. 112, 2339 (2014).

12.J. Rozmus, J.-C. de Hemptinne, A. Galindo, S. Dufal, and P. Mougin, Ind. Eng. Chem. Res. 52, 9979 (2013).

${ }^{13}$ S. Ahmed, N. Ferrando, J.-C. de Hemptinne, J.-P. Simonin, O. Bernard, and O. Baudouin, Fluid Phase Equilib. 459, 138 (2018). 
14Z. Liu, W. Wang, and Y. Li, Fluid Phase Equilib. 227, 147 (2005).

${ }^{15}$ H. Zhao, M. C. dos Ramos, and C. McCabe, J. Chem. Phys. 126, 244503 (2007).

${ }^{16}$ S. Herzog, J. Gross, and W. Arlt, Fluid Phase Equilib. 297, 23 (2010).

${ }^{17}$ L. Blum, J. Chem. Phys. 61, 2129 (1974).

${ }^{18}$ L. Blum and D. Wei, J. Chem. Phys. 87, 555 (1987).

${ }^{19}$ L. Blum, F. Vericat, and W. Fawcett, J. Chem. Phys. 96, 3039 (1992).

${ }^{20}$ J. Wu and J. M. Prausnitz, Ind. Eng. Chem. Res. 37, 1634 (1998).

${ }^{21}$ R. Inchekel, J.-C. De Hemptinne, and W. Fürst, Fluid Phase Equilib. 271, 19 (2008).

${ }^{22}$ L. Sun, X. Liang, N. von Solms, and G. M. Kontogeorgis, Fluid Phase Equilib. (2018), https://doi.org/10.1016/j.fluid.2018.12.033, accepted manuscript.

${ }^{23}$ H.-G. Simon, H. Kistenmacher, J. M. Prausnitz, and D. Vortmeyer, Chem. Eng. Process. 29, 139 (1991).

${ }^{24}$ F. Ball, W. Fürst, and H. Renon, AIChE J. 31, 392 (1985).

${ }^{25}$ Y. Liu and S. Watanasiri, Fluid Phase Equilib. 116, 193 (1996).

${ }^{26}$ J. Vincze, M. Valiskó, and D. Boda, J. Chem. Phys. 133, 154507 (2010).

${ }^{27}$ M. Valiskó and D. Boda, J. Chem. Phys. 140, 234508 (2014).

${ }^{28}$ W. G. McMillan and J. E. Mayer, J. Chem. Phys. 13, 276 (1945), see page 295 for details about this point.

${ }^{29}$ X. Ji, S. P. Tan, H. Adidharma, and M. Radosz, Ind. Eng. Chem. Res. 44, 7584 (2005).

${ }^{30}$ S. Müller, A. G. de Castilla, C. Taeschler, A. Klein, and I. Smirnova, Fluid Phase Equilib. 483, 165 (2019).

${ }^{31}$ H. S. Harned and B. B. Owen, The physical chemistry of electrolytic solutions (Reinhold Pub. Corp., New York, 1958) p. 81.

${ }^{32}$ M. Born, Z.Phys. 1, 45 (1920).

${ }^{33}$ J. Barthel, R. Buchner, and M. Münsterer, in Electrolyte Data Collection, Vol. 12 (DECHEMA, Frankfurt am Main, 1995).

${ }^{34}$ D. Wei and L. Blum, J. Chem. Phys. 87, 2999 (1987).

${ }^{35}$ L. Blum and W. Fawcett, J. Phys. Chem. 96, 408 (1992).

36F. Garisto, P. G. Kusalik, and G. N. Patey, J. Chem. Phys. 79, 6294 (1983).

${ }^{37}$ W. R. Fawcett, J. Phys. Chem. B 103, 11181 (1999).

${ }^{38}$ I. Rips and M. Tachiya, J. Chem. Phys. 107, 3924 (1997).

${ }^{39}$ J.-P. Simonin, J. Chem. Phys. 134, 054508 (2011). 
${ }^{40}$ I. Y. Shilov and A. K. Lyashchenko, J. Phys. Chem. B 119, 10087 (2015).

${ }^{41} \mathrm{R}$. Pottel, in Water: A Comprehensive Treatise, Vol. 3. Aqueous Solutions of Simple Electrolytes, edited by F. Franks (Springer US, Boston, MA, 1973) pp. 401-431.

${ }^{42}$ W. J. Hamer and Y.-C. Wu, J. Phys. Chem. Ref. Data 1, 1047 (1972).

${ }^{43}$ J. E. Huheey, E. A. Keiter, R. L. Keiter, and O. K. Medhi, Inorganic chemistry: principles of structure and reactivity (Pearson Education India, 2006).

${ }^{44}$ Y.-Z. Wei, P. Chiang, and S. Sridhar, J. Chem. Phys. 96, 4569 (1992).

${ }^{45}$ D. P. Fernández, Y. Mulev, A. Goodwin, and J. L. Sengers, J. Phys. Chem. Ref. Data 24, 33 (1995).

${ }^{46}$ O. Söhnel and P. Novotnỳ, in Physical sciences data, Vol. 22 (Elsevier Publishing Company, 1985).

${ }^{47}$ L. Blum, J. Stat. Phys. 18, 451 (1978).

${ }^{48}$ Z.-P. Liu, Y.-G. Li, and J.-F. Lu, J. Phys. Chem. B 106, 5266 (2002).

${ }^{49}$ G. S. Rushbrooke, G. Stell, and J. Høye, Mol. Phys. 26, 1199 (1973).

${ }^{50}$ J. S. Høye and E. Lomba, J. Chem. Phys. 88, 5790 (1988).

${ }^{51}$ J.-P. Simonin, L. Blum, and P. Turq, J. Phys. Chem. 100, 7704 (1996).

${ }^{52}$ J. Salacuse and G. Stell, J. Chem. Phys. 77, 3714 (1982).

${ }^{53}$ J.-P. Simonin, J. Phys. Chem. 99, 1577 (1995).

${ }^{54}$ T. Boublik, J. Chem. Phys. 53, 471 (1970).

${ }^{55}$ N. F. Carnahan and K. E. Starling, J. Chem. Phys. 51, 635 (1969).

${ }^{56}$ S. A. Adelman, J. Chem. Phys. 64, 724 (1976).

${ }^{57}$ M. Wertheim, J. Chem. Phys. 55, 4291 (1971).

${ }^{58}$ R. Robinson and R. Stokes, Electrolyte Solutions (Butterworths, 1968).

${ }^{59}$ C. J. F. Böttcher, O. C. van Belle, P. Bordewijk, and A. Rip, Theory of electric polarization, Vol. 1 (Elsevier, 1973).

${ }^{60}$ D. Y. C. Chan, D. J. Mitchell, and B. W. Ninham, J. Chem. Phys. 70, 2946 (1979).

${ }^{61}$ A. Voet, Trans. Faraday Soc. 32, 1301 (1936).

${ }^{62}$ J. D'Ans, H. Surawski, and C. Synowietz, in Landolt-Börnstein database: Numerical Data and Functional Relationships in Science and Technology. Group IV: Macroscopic and Technical Properties of Matter, Vol. 1 (Springer Berlin, Heidelberg, New York, 1977).

${ }^{63}$ H. Berendsen, J. Grigera, and T. Straatsma, J. Phys. Chem. 91, 6269 (1987).

${ }^{64}$ C. A. Coulson and D. Eisenberg, Proc. Roy. Soc. Lond. A 291, 445 (1966). 
${ }^{65}$ A. V. Gubskaya and P. G. Kusalik, J. Chem. Phys. 117, 5290 (2002).

${ }^{66}$ L. D. Site, A. Alavi, and R. Lynden-Bell, Mol. Phys. 96, 1683 (1999).

${ }^{67}$ P. L. Silvestrelli and M. Parrinello, Phys. Rev. Lett. 82, 3308 (1999).

${ }^{68}$ P. Schatzberg, J. Phys. Chem. 71, 4569 (1967).

${ }^{69}$ R. A. Robinson and H. S. Harned, Chem. Rev. 28, 419 (1941).

${ }^{70}$ C. Chan, N. Tioh, and G. Hefter, Polyhedron 3, 845 (1984).

${ }^{71}$ R. Buchner, G. T. Hefter, and J. Barthel, J. Chem. Soc., Faraday Trans. 90, 2475 (1994).

${ }^{72}$ K.-L. Hsia and R. M. Fuoss, J. Am. Chem. Soc. 90, 3055-(1968)

${ }^{73}$ H. S. Dunsmore, S. K. Jalota, and R. Paterson, J. Chem. Soc., Faraday Trans. I 68, 1583 (1972).

${ }^{74}$ H. McKay, Trans. Faraday Soc. 52, 1568 (1956).

${ }^{75}$ J.-P. Simonin, O. Bernard, and L. Blum, J.Phys. Chem. B 102, 4411 (1998).

${ }^{76}$ A. H. Harvey, J. Chem. Phys. 95, 479 (1991).

${ }^{77}$ C. P. Kelly, C. J. Cramer, and D. G. Truhlar, J. Phys. Chem. B 110, 16066 (2006).

${ }^{78} \mathrm{~S}$. Cummings, J. Enderby, G. W. Neilson, J. Newsome, R. Howe, W. Howells, and A. Soper, Nature 287, 714 (1980).

${ }^{79}$ R. Mancinelli, A. Botti, F. Bruni, M. A. Ricci, and A. K. Soper, J. Phys. Chem. B 111, 13570 (2007). 


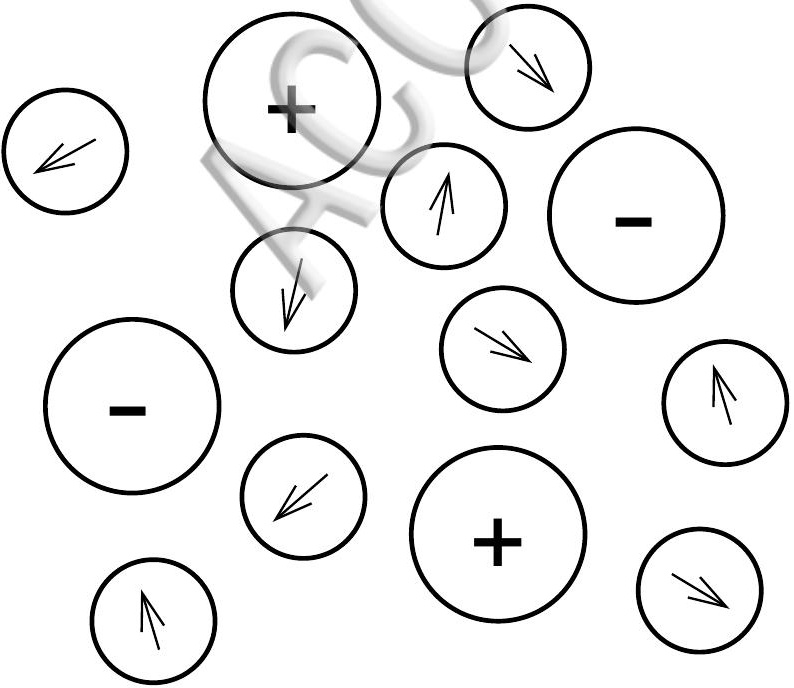




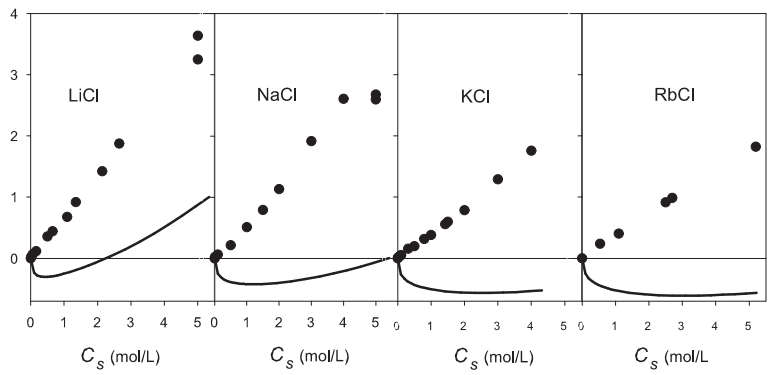




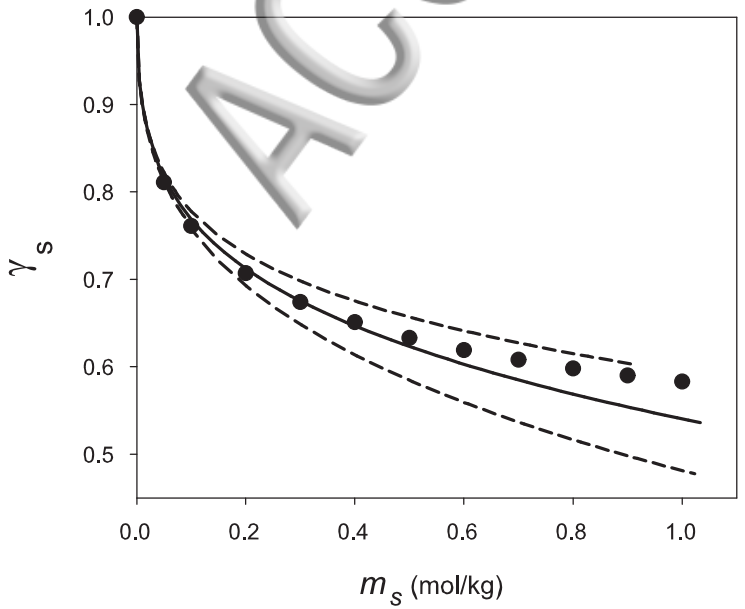




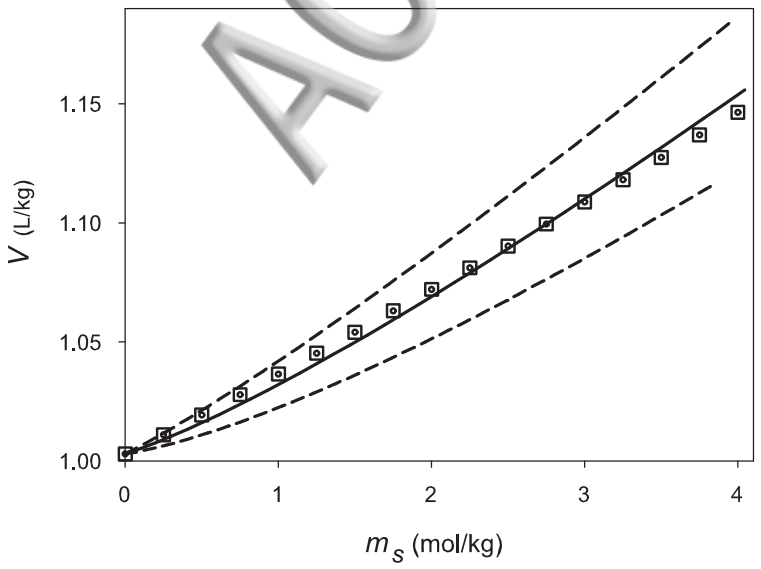




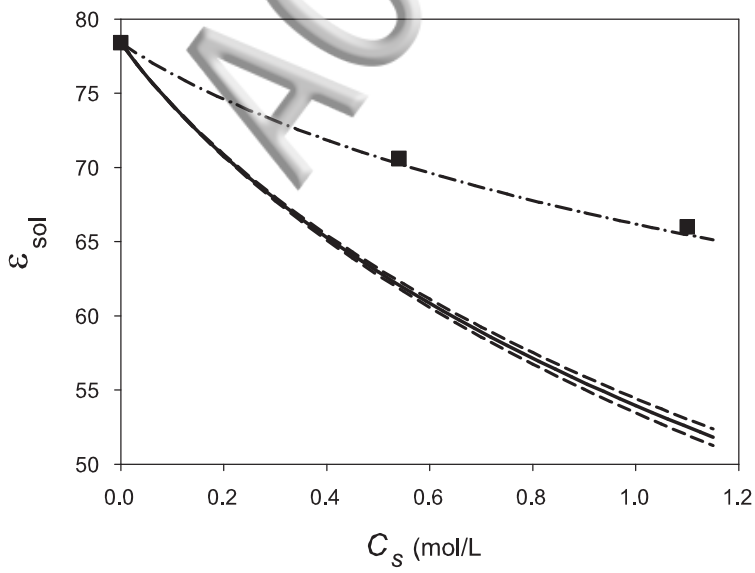




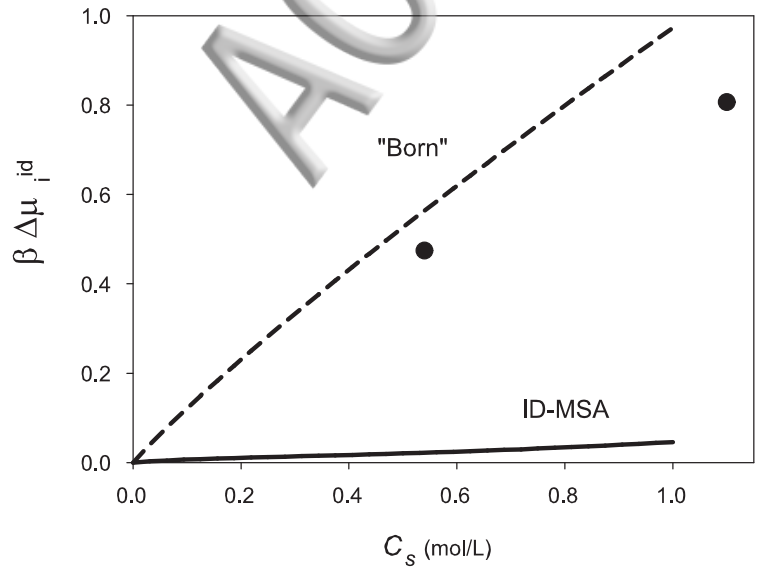




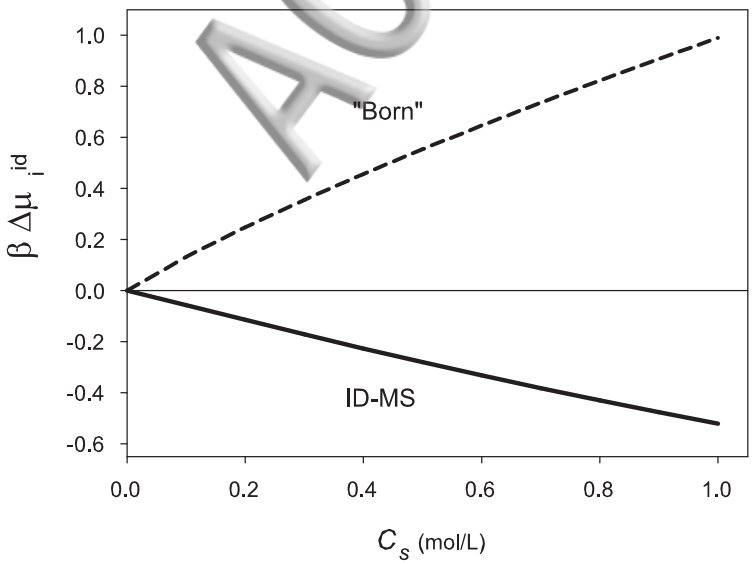


